DRAFT VERSION MARCH 17, 2022

Preprint typeset using LTEX style emulateapj v. 12/16/11

\title{
SHORT-TERM RADIO VARIABILITY AND PARSEC-SCALE STRUCTURE IN A GAMMA-RAY NARROW-LINE SEYFERT 1 GALAXY 1H 0323+342
}

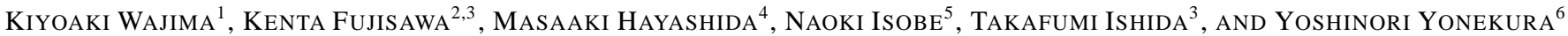

Draft version March 17, 2022

\begin{abstract}
We made simultaneous single-dish and very long baseline interferometer (VLBI) observations of a narrow-line Seyfert 1 galaxy (NLS1) 1H 0323+342, showing gamma-ray activity revealed by Fermi/LAT observations. We found significant variation of the total flux density at $8 \mathrm{GHz}$ on the time scale of one month by the single-dish monitoring. The total flux density varied by $5.5 \%$ in 32 days, which is comparable to the gamma-ray variability time scale, corresponding to the variability brightness temperature of $7.0 \times 10^{11} \mathrm{~K}$. The source consists of central and southeastern components on the parsec (pc) scale. The flux of only the central component decreased in the same way as the total flux density, indicating that the short-term radio variability, and probably the gammaray emitting region, is associated with this component. From the VLBI observations we obtained the brightness temperatures of greater than $(5.2 \pm 0.3) \times 10^{10} \mathrm{~K}$, and derived the equipartition Doppler factor of greater than 1.7 , the variability Doppler factor of 2.2 , and the $8 \mathrm{GHz}$ radio power of $10^{24.6} \mathrm{~W} \mathrm{~Hz}^{-1}$. Combining them we conclude that acceleration of radio jets and creation of high-energy particles are ongoing in the central engine, and that the apparent very radio-loud feature of the source is due to the Doppler-boosting effect, resulting in the intrinsic radio loudness to be an order of magnitude smaller than the observed values. We also conclude that the pc-scale jet represents recurrent activity from the the spectral fitting and the estimated kinematic age of pc- and kpc-scale extended components with different position angle.
\end{abstract}

Keywords: galaxies: active — galaxies: individual $(1 \mathrm{H} 0323+342)$ — galaxies: Seyfert — radio continuum: galaxies - techniques: interferometric

\section{INTRODUCTION}

Gamma-ray emission from active galactic nuclei (AGNs) is one of the important properties in terms of activities and energetics of the central engine and emergence of relativistic jets. In the EGRET (Energetic Gamma-Ray Experiment Telescope) era, nearly a hundred of gamma-ray emitting AGNs were known (Hartman et al. 1999; Sowards-Emmerd et al. 2003, 2004). Those are predominantly radio-loud AGNs and categorized as blazars, suggesting a close connection between gamma-ray and radio emission. On the other hand, observations with the Fermi Gamma-ray Space Telescope (hereafter Fermi) have resulted in the identification of more than a thousand AGNs, some of which are categorized as non-blazar active galaxies or radio galaxies (Nolan et al. 2012). Moreover, Fermi has also revealed the existence of new classes of gamma-ray emitting AGNs, one of which is narrow-line Seyfert 1 galaxy (NLS1). NLS1 is a subclass of AGNs and identified by their optical properties; narrow permitted lines, $\operatorname{FWHM}(\mathrm{H} \beta)<2000 \mathrm{~km} \mathrm{~s}^{-1}$, emitted from the broad line region, $[\mathrm{O} \mathrm{III}] / \mathrm{H} \beta<3$, and a bump due to Fe II

\footnotetext{
${ }^{1}$ Shanghai Astronomical Observatory, Chinese Academy of Sciences, 80 Nandan Road, Xuhui District, Shanghai 200030, China; kwajima@shao.ac.cn

${ }^{2}$ The Research Institute for Time Studies, Yamaguchi University, 1677 1 Yoshida, Yamaguchi, Yamaguchi 753-8511, Japan

${ }^{3}$ Graduate School of Science and Engineering, Yamaguchi University, 1677-1 Yoshida, Yamaguchi, Yamaguchi 753-8512, Japan

${ }^{4}$ Institute for Cosmic Ray Research, The University of Tokyo, 5-1-5 Kashiwanoha, Kashiwa, Chiba 277-8582, Japan

${ }^{5}$ The Institute of Space and Astronautical Science, Japan Aerospace Exploration Agency, 3-1-1 Yoshinodai, Chuo-ku, Sagamihara, Kanagawa 252-5210, Japan

${ }^{6}$ Center for Astronomy, Ibaraki University, 2-1-1 Bunkyo, Mito, Ibaraki 310-8512, Japan
}

(Osterbrock \& Pogge 1985; Pogge 2000).

First detection of gamma-ray emission in a radio-loud NLS1 by Fermi was made for PMN J0948+0022 (Abdo et al. 2009a). Intensive observations by the Large Area Telescope (LAT) onboard Fermi have revealed new detections of gamma-rays of other three radio-loud NLS1s (Abdo et al. 2009b). Seven NLS1s are listed as gamma-ray sources detected by Fermi/LAT to date (Foschini 2011) and the number of gamma-ray NLS1s is increasing7. These are surprising discoveries because most of NLS1s are hosted in a spiral galaxy, which usually does not have relativistic jets, while blazars are hosted in an elliptical galaxy. Although milliarcsecond (mas)-scale images have been obtained for several gammaray NLS1s with very long baseline interferometer (VLBI) observations (Doi et al. 2006b, 2007; Giroletti et al. 2011; Linford et al. 2012; Orienti et al. 2012; D'Ammando et al. 2012, 2013) and some of them have revealed presence of a closely aligned relativistic jet, there are still large uncertainties for the derived parameters in gamma-ray NLS1s and it is therefore important to investigate the parsec (pc)-scale properties.

NLS1s are also considered to have high-mass accretion rates, close to the Eddington limit, and lower black hole masses $\left(M_{\mathrm{BH}} \sim 10^{5-7} M_{\odot}\right)$ compared to other classes of AGNs (Boroson 2002). Considering the relation among radio loudness, the black hole mass, and the accretion rate (Greene et al. 2006: Zhou et al. 2007, and references therein), those properties imply that NLS1s are generally radio-quiet objects and have weak or no jet activity (Maccarone et al. 2003). In fact, previous studies show that the fraction of radio-loud NLS1s is low (about 7\%) compared to other AGN classes

\footnotetext{
${ }^{7}$ See http://www.brera.inaf.it/utenti/foschini/gNLS1/catalog.html
} 
(Komossa et al. 2006) and NLS1s have lower jet activities compared to other types of radio-loud AGNs (Greene et al. 2006; Zhou et al. 2006). NLS1s are thus considered to be at the high/soft state containing a slim disk while blazars and flat-spectrum radio quasars are at the low/hard state, in the analogy of Galactic X-ray binaries (Mineshige et al. 2000; Maccarone et al. 2003). It is interesting to study relation between the state transition and the jet activity in AGNs since we can import knowledge of the state transition for Galactic Xray binaries to AGNs. Again, multiepoch and high-resolution radio observations are essential to investigate them.

In this paper we report the results of simultaneous singledish and very long baseline interferometer (VLBI) observations of $1 \mathrm{H} 0323+342$ at $8 \mathrm{GHz}$, including the archival data obtained by the Very Long Baseline Array (VLBA). $1 \mathrm{H} 0323+342$ is one of seven gamma-ray emitted NLS1s detected by Fermi/LAT (Abdo et al. 2009b). An optical observation by the Hubble Space Telescope shows that the source is hosted in a spiral galaxy containing single spiral arm (Zhou et al. 2007), while another results claim that the host galaxy of the source has a ring structure triggered by an interacting/merging process (Antón et al. 2008). $M_{\mathrm{BH}}$ is estimated to be a few times $10^{7} M_{\odot}$ with a few different methods using the width and luminosity of the $\mathrm{H} \beta$ line and the continuum luminosity at $5100 \AA$ (Zhou et al. 2007). This is in good agreement with $M_{\mathrm{BH}}=10^{7.0} M_{\odot}$ from the parameter used to model the spectral energy distribution (Abdo et al. 2009b). Abdo et al. (2009b) estimate the accretion disk luminosity to be $1.4 \times 10^{45} \mathrm{erg} \mathrm{s}^{-1}$, or $0.9 L_{\mathrm{Edd}}$, where $L_{\mathrm{Edd}}$ is the Eddington luminosity. Although these characteristics are typically seen among NLS1s, the source has extremely high radio loudness $R_{1.4}=f_{1.4 \mathrm{GHz}} / f_{440 \mathrm{~nm}}=318$ (Foschini 2011), or $R_{5}=f_{5 \mathrm{GHz}} / f_{440 \mathrm{~nm}}=246$ (Doi et al. 2012), and is listed in one of 29 very radio-loud $(R>100)$ NLS1s (Foschini 2011), implying activities due to relativistic jets in the inner region. A redshift, $z$, of $0.0629 \pm 0.0001$ is measured by Zhou et al. (2007) and we adopt this value, although some of previous articles adopt $z=0.061$ reported by Marchã et al. (1996). $1 \mathrm{H} 0323+342$ is the nearest object among gamma-ray NLS1s, allowing us to investigate the inner region of the source with higher angular resolution.

The content of the paper is as follows. Our observations and data reduction procedures are described in Section 2 . We present the results of single-dish and VLBI observations in Section 3 In Section 4 we describe the results of the archival VLBA observations. In Section 5, we discuss the observed variability feature, relation to general properties of NLS1s, and possible connections between gamma-ray emission and the pc-scale structure. The achievements of our study are summarized in Section 6. Throughout this paper, we define the spectral index, $\alpha$, as $S_{\nu} \propto \nu^{+\alpha}$, where $S_{\nu}$ is the flux density at the frequency $\nu$, and we adopt a $\Lambda$ CDM cosmology with $H_{0}=71 \mathrm{~km} \mathrm{~s}^{-1} \mathrm{Mpc}^{-1}, \Omega_{\Lambda}=0.73$, and $\Omega_{\mathrm{M}}=0.27$ from the results of the Wilkinson Microwave Anisotropy Probe (Komatsu et al. 2009), corresponding to an angular-to-linear scale conversion of $1.20 \mathrm{pc} \mathrm{mas}^{-1}$ for $1 \mathrm{H} 0323+342$.

\section{OBSERVATIONS AND DATA REDUCTION}

The total flux density of $1 \mathrm{H} 0323+342$ was monitored by Yamaguchi $32 \mathrm{~m}$ radio telescope (hereafter Y32; Fuijisawa et al. 2002) at 25 epochs from 2010 November 9 (2010.858) to 2011 February 5 (2011.099). The Y32 observations were made typically every two or three days. The monitoring was done at $8.38 \mathrm{GHz}$ with the total bandwidth of $400 \mathrm{MHz}$ in the total power mode. We employed the 'Z-scan' method to remove both the gain and atmospheric fluctuations, and the pointing offsets. The detailed procedure of the observation and data reduction of the method is described by Kadota et al. (2012). The flux density of $1 \mathrm{H} 0323+342$ was measured by comparing the total received power with that of a strong, nearby radio source 3C 123 (9.310 Jy; Ott et al.1994).

Observations with the Japanese VLBI Network (JVN; Doi et al. 2006a) were made simultaneously with Y32 monitoring at three epochs in November 2010. The observation dates at each epoch are shown in Table 1. Six radio telescopes, VERA (the VLBI Exploration of Radio Astrometry; Kobayashi et al. 2003) 4 telescopes with the antenna diameter of $20 \mathrm{~m}$ each, Hitachi $32 \mathrm{~m}$ telescope (Yonekura et al. 2013), and Kashima 34 m telescope, were used for the observations, while Kashima did not participate in epoch 1. Observations were made in right-circular polarization at $8.424 \mathrm{GHz}$ with the total bandwidth of $32 \mathrm{MHz}$. Observed sources were $1 \mathrm{H} 0323+342$ as a target, $\mathrm{J} 0310+3814$ as a gain calibrator (separation angle of 4.94 from $1 \mathrm{H} 0323+342$ ), DA 193 as a flux calibrator, and 3C 84 as a bandpass calibrator. The total on-source duration for $1 \mathrm{H} 0323+342$ is 443 minutes in all epochs. The data were correlated using the Mitaka FX correlator (Shibata et al. 1998) with the output preaveraging time of 2 seconds.

The JVN data were reduced using the Astronomical Image Processing System (AIPS) software (Greisen 2003) for amplitude and phase calibration, and the Caltech software Difmap (Shepherd 1997) for imaging and self-calibration. We did not employ a standard a priori amplitude calibration using the system noise temperature because it was not measured during the observations at several stations. Flux densities of each source were therefore determined as follows. We determined the flux density of DA 193 by single-dish observations with Y32 to be $4.787 \pm 0.027 \mathrm{Jy}$ on 2010 November 15 and $4.907 \pm 0.040 \mathrm{Jy}$ on 2010 November 29. We assumed the correlated flux density of DA 193 with JVN to be same as that with single-dish measurements since DA 193 can be considered as a point source within the JVN baseline lengths. Flux densities were determined comparing cross-correlation amplitudes for each source with those for DA 193. To correct variation of the antenna gain due to change of the antenna elevation, we applied self-calibration to the gain calibrator $\mathrm{J} 0310+3814$. It can be considered as a point source and was observed once an hour with the on-source duration of 5 minutes per scan. A fitted curve to the solutions for the gain calibrator was applied to other sources.

Through the procedure described above, we determined flux densities of J0310+3814 at epochs 2 and 3 to be comparable to previous results by VLBI observations at the same frequency. On the other hand, the flux density of J0310+3814 at epoch 1, obtained by the AIPS task GETJY, was determined to be twice $(1.913 \mathrm{Jy})$ than that at epochs $2(0.985 \mathrm{Jy})$ and $3(0.989 \mathrm{Jy})$. We finally determined a flux density of $\mathrm{J} 0310+3814$ at epoch 1 to be same as that obtained at epoch 2 for the following reasons. At epochs 2 and 3, both Kashima $34 \mathrm{~m}$ and Hitachi $32 \mathrm{~m}$ telescopes participate in the observations. Those antennas have system equivalent flux densities (SEFDs) to be approximately 7 times better than those of VERA 4 antennas at $8 \mathrm{GHz}$. We therefore consider the results at epochs 2 and 3 to be more likely than that at epoch 1 . In addition, we obtain similar flux densities at epochs 2 and 3 as mentioned above. We therefore consider that $\mathrm{J} 0310+3814$ 
does not show variability during the JVN campaign.

We exported the calibrated visibility data to Difmap for imaging. The amplitude calibration error obtained by selfcalibration procedure was $10 \%, 4 \%$, and $4 \%$ for each epoch. To ensure a better angular resolution, we adopt uniform weighting of the data with gridding weights scaled by amplitude errors raised to the power of -1 . The maximum and minimum projected baseline lengths of the JVN observation were 2,290 km or $64 \mathrm{M} \lambda$ (VERA Mizusawa - VERA Ishigakijima baseline) and $77 \mathrm{~km}$ or $2.2 \mathrm{M} \lambda$ (Hitachi - Kashima baseline), respectively.

\section{RESULTS}

\subsection{Single-Dish Monitoring with Yamaguchi $32 \mathrm{~m}$ Radio Telescope}

Figure 1 shows the total flux density measurements of $1 \mathrm{H} 0323+342$ by Y32. Numerical data of the measurements are shown in Table 2. Error bars at each point in Figure 1 indicate a $1 \sigma$ standard deviation divided by square root of the number of independent measurements. The source has also been monitored between 2010.6 to 2011.2 at nine frequencies from 2.64 to $142 \mathrm{GHz}$ by the F-GAMMA Program (Fuhrmann et al. 2011; Angelakis et al. 2012). Our results are in good agreement with theirs at $8.35 \mathrm{GHz}$ within the margin of error. F-GAMMA results show that the flux density varies almost simultaneously at observing frequencies from $2.64 \mathrm{GHz}$ to $32.0 \mathrm{GHz}$ (Fuhrmann et al. 2011), therefore we believe that the flux variation observed with Y32 is intrinsic. The total flux varies significantly during the period of Y32 monitoring compared with the constant flux. The maximum and minimum flux densities during Y32 monitoring can be seen as $432 \pm 24 \mathrm{mJy}$ on 2010 November 13 and $266 \pm 30 \mathrm{mJy}$ on 2011 February 5, respectively, corresponding to a flux decrease of $38 \%$ in 84 days, while the total flux does not decrease monotonically. We apply a third-order polynomial to all measurements of Y32 to estimate the local minimum and maximum of the flux densities (reduced $\chi^{2}=3.61$ with d.o.f. of 23 , and 2.33 with d.o.f. of 21 for linear and cubic function, respectively, which is significant at $>99.9 \%$ confidence level). The local minimum of $325 \mathrm{mJy}$ at the epoch 2010.956 and the local maximum of $344 \mathrm{mJy}$ at the epoch 2011.043 are derived from the best-fit curve, corresponding to the flux variation of $5.5 \%$ in 32 days. While Fuhrmann et al. (2011) pointed out that the source indicates variability on time scales of months to years, our results clearly show the existence of the short-term radio variability on the time scale of one month. Moreover, this is comparable to the (e-folding) gamma-ray variability time scale of $17.7 \pm 14.4$ days with the two-year monitoring results from 2008 September to 2010 September by Fermi/LAT (Calderone et al. 2011), suggesting that the source of short-term radio variability is probably associated with the gamma-ray emitting region.

\subsection{JVN Observation Results}

Figure 2 shows images of $1 \mathrm{H} 0323+342$ at each epoch. The image parameters are shown in Table 1 in addition to the total flux density of all CLEAN components. JVN images could not resolve the central one, containing a few components in the images obtained by VLBA, as shown in Section 4 . To quantify the relative location and the flux density of each component of the JVN images, we modeled the calibrated images with elliptical Gaussian components. Table 3 shows the model fitting results obtained by the AIPS task JMFIT.
The formal errors of each parameter are estimated using the fomulae from Fomalont (1999). The data at all epochs are modeled satisfactorily by two distinct components, brighter and unresolved component labeled $\mathrm{C}$ and weaker component labeled D1, situated to the southeast of $\mathrm{C}$. The angular size of $\mathrm{C}$ shown in Table 3 is therefore the upper limit. The sum of the flux densities of the components C and D1 is in good agreement with both the total CLEANed flux and single-dish measurements by Y32, as shown in Tables 1 and 2, while the Very Large Array (VLA) observations at $1.4 \mathrm{GHz}$ on 2006 February 7 and 2007 January 15 (VLA observation code; AP501) show the source having a large-scale structure with the total size of about a hundred kpc (Antón et al. 2008) and with the flux density for the extended component of $198 \mathrm{mJy}$ (Doi et al. 2012). We consider that the extended structure has an optically thin spectrum and therefore shows very weak emission at around $8 \mathrm{GHz}$. It should also be noted that the flux density of the component $\mathrm{C}$ gradually decreases similar to the total flux density, while the flux density of the component D1 seems to be stable, as shown in Figure 1. The results indicate that the short-term radio variability observed with Y32 (and probably the gamma-ray emitting region) is mainly associated with the central component.

\section{VLBA ARCHIVE DATA}

To investigate time variation of the pc-scale structure of the source, we reduced data of the archival VLBA observations at 2, 8, and $15 \mathrm{GHz}$. The observations are summarized in Table 4. The simultaneous VLBA observation at 2.3 and $8.3 \mathrm{GHz}$ on 1996 May 16 has been published by Beasley et al. (2002) (VLBA observation code; BB023). The $15 \mathrm{GHz}$ observation has been carried out as part of the VLBA MOJAVE Program (Lister et al. 2009) on 2010 October 15, just half a month before the JVN epoch 1 observation. Figure 3 shows images of $1 \mathrm{H} 0323+342$ with VLBA. Descriptions of each image are shown in Table 4 The size of restoring beam for the BK077 observation is slightly larger than those for other observations at $8 \mathrm{GHz}$ since the visibilities with Mauna Kea station, which gives longer baseline data, are excluded because of inadequate accuracy of the amplitude calibration. All images shown in Figure 3 have similar pc-scale structure to those of the JVN observations, consisting of two distinct components, while each of them are slightly resolved. We modeled the calibrated VLBA images with elliptical Gaussian components. Table 5 shows the model fitting results. The model-fitting procedure and estimation of errors for each parameter are made in the same manner with those for the JVN data. We could reconstruct VLBA images with three elliptical Gaussian components $\mathrm{C} 0$, D2, and D1 at $8 \mathrm{GHz}$, while the component D1 could be resolved into two components D1b and D1a in the epochs 2005.636 and 2008.303. In the epochs 1996.374 at $2.3 \mathrm{GHz}$ and 2003.688 the images could be modeled by two components because of lower angular resolution than other epochs. The component $\mathrm{C}$ in the JVN images was resolved into two components $\mathrm{C} 0$ and $\mathrm{D} 2$ in the VLBA images at $8 \mathrm{GHz}$. For the $15 \mathrm{GHz}$ image we could find an additional component $\mathrm{D} 3$ in the vicinity of $\mathrm{C} 0$.

We also compared the image of JVN epoch 1 with that of the $15 \mathrm{GHz}$ VLBA. We restored the synthesized beam of the VLBA $15 \mathrm{GHz}$ image to the same size with the JVN epoch 1 observation and obtained the flux density of the central component as $276 \mathrm{mJy}$. The spectral index of $\alpha_{8}^{15}=-0.70$ is thus

\footnotetext{
${ }^{8}$ See also http://www.physics.purdue.edu/MOJAVE/
} 
derived from the observations by JVN epoch 1 and the VLBA MOJAVE. The radio power at a rest frequency of $8.0 \mathrm{GHz}$ is estimated to be $P_{8 \mathrm{GHz}}=10^{24.6} \mathrm{~W} \mathrm{~Hz}^{-1}$, in which a $k$-correction is applied using $\alpha_{8}^{15}$.

\section{DISCUSSION}

\subsection{Brightness Temperature}

We have two kinds of radio observations, a single-dish monitoring and VLBI observations, therefore we can derive brightness temperatures, $T_{\mathrm{B}}$, using different ways from these results. $T_{\mathrm{B}}$ in the source's rest frame can be obtained with an image as

$$
T_{\mathrm{B}, \text { rest }}^{\text {(image) }}=1.77 \times 10^{9}(1+z) \frac{S_{\nu}}{\nu^{2} \theta_{\text {maj }} \theta_{\text {min }}} \quad[\mathrm{K}],
$$

where $\theta_{\text {maj }}$ [mas] and $\theta_{\text {min }}$ [mas] are the FWHM sizes of the Gaussian component in the major and minor axes, respectively, and $S_{\nu}[\mathrm{mJy}]$ is the flux density at an observing frequency $\nu[\mathrm{GHz}]$. Given the model fitting result of $S_{\nu}=419 \mathrm{mJy}, \theta_{\text {maj }}<1.12 \mathrm{mas}$, and $\theta_{\text {min }}<0.12$ mas for the component $\mathrm{C}$ at epoch 1 which leads the highest $T_{\mathrm{B}}$ among our JVN observations, we obtain $T_{\mathrm{B} \text {,rest }}^{\text {(imag) }}>(8.3 \pm 1.3) \times 10^{10} \mathrm{~K}$. $T_{\mathrm{B} . \text { rest }}^{\text {(image) }}$ at the other epochs are shown in Table 3 , Linford et al. (2012) made a VLBA observation of the source on 2010 June 30 at $4.8 \mathrm{GHz}$ and estimated the brightness temperature of the core component to be $2.15 \times 10^{10} \mathrm{~K}$, which is comparable to our results.

We can also obtain $T_{\mathrm{B}}$ with the flux variation as

$$
T_{\mathrm{B}, \text { rest }}^{(\mathrm{var})}=4.1 \times 10^{10}\left[\frac{D_{\mathrm{L}}}{\Delta t(1+z)}\right]^{2} \frac{\Delta S_{\nu}}{\nu^{2}}
$$

(Wagner \& Witzel 1995), where $D_{\mathrm{L}}[\mathrm{Mpc}]$ is the luminosity distance to the source, $\Delta S_{\nu}$ [mJy] is a change in the observed flux density at an observing frequency $\nu[\mathrm{GHz}]$ during a period of $\Delta t$ [days]. Given the single-dish monitoring result of $\Delta S_{\nu}=19 \mathrm{mJy}, \Delta t=32$ days, and applying $D_{\mathrm{L}}=270 \mathrm{Mpc}$, we obtain $T_{\mathrm{B}, \text { rest }}^{(\mathrm{var})}=7.0 \times 10^{11} \mathrm{~K}$. Alternatively $T_{\mathrm{B}, \text { rest }}^{(\mathrm{var})}$ can be estimated by applying the maximum and minimum flux densities during Y32 monitoring, $\Delta S_{\nu}=166 \mathrm{mJy}, \Delta t=84$ days, to be $T_{\mathrm{B} \text {,rest }}^{(\mathrm{var})}=8.9 \times 10^{11} \mathrm{~K}$, which is in good agreement with the above result.

The brightness temperature has been measured for several gamma-ray NLS1s with radio observations. Zhou et al. (2003) analyzed the VLA observation data of a quasar PMN J0948+0022 known to have the highest radio loudness in NLS1s, and found the long-term flux variation with the time scale of a few years. The brightness temperature was estimated to be $\sim 10^{13} \mathrm{~K}$ from the variability. This high $T_{\mathrm{B}}$ was confirmed by Doi et al. (2006b) with their VLBA images and flux variation at $1.7-15.4 \mathrm{GHz}$, which result in $T_{\mathrm{B} \text {,rest }}^{\text {(image) }}>5.5 \times 10^{11} \delta^{-1} \mathrm{~K}$ and $T_{\mathrm{B}, \text { rest }}^{\text {(var) }}>3.3 \times 10^{13} \delta^{-3} \mathrm{~K}$, where $\delta$ is the Doppler factor. $T_{\mathrm{B}}$ of PMN J0948+0022 was also measured with the global e-VLBI observation at $22 \mathrm{GHz}$ to be $T_{\mathrm{B} . \text { rest }}^{\text {(image }} \sim 3.4 \times 10^{11} \mathrm{~K}$ (Giroletti et al. 2011). Yuan et al. (2008) compiled previous radio observations for a sample of radio-loud NLS1s and estimated $T_{\mathrm{B}, \text { rest }}^{(\mathrm{var})}$ for three gammaray sources; $1.1 \times 10^{13} \mathrm{~K}$ for SBS $0846+513,5.1 \times 10^{12} \mathrm{~K}$ for PMN J0948+0022, and $3.6 \times 10^{12} \mathrm{~K}$ for PKS $1502+036$. D'Ammando et al. (2013) found significant flux variation by both single-dish flux monitoring with the Owens Valley Radio Observatory $40 \mathrm{~m}$ telescope at $15 \mathrm{GHz}$ and multiepoch VLA observations at eight frequencies from 1.4 to $22.2 \mathrm{GHz}$, and estimated $T_{\mathrm{B} \text {,rest }}^{\text {(var) }}$ to be $2.5 \times 10^{13} \mathrm{~K}$ from single-dish measurements. Although our $T_{\mathrm{B}}$ is a few orders of magnitude lower than those mentioned above, it still exceeds the upper limit of $T_{\mathrm{B}}$ assuming the condition of energy equipartition, as shown in Section 5.2, suggesting that acceleration of the radio jet is ongoing in the central engine of the source.

The $8 \mathrm{GHz}$ radio power is estimated to be $P_{8 \mathrm{GHz}}=$ $10^{24.6} \mathrm{~W} \mathrm{~Hz}^{-1}$, as shown in Section 4 which is much higher than the radio powers at $1.5 \mathrm{GHz}$ of up to $10^{23.7} \mathrm{~W} \mathrm{~Hz}^{-1}$ measured in a sample of the most radio-luminous starburst galaxies (Lonsdale et al.1993; Smith et al. 1998a b). This also supports that not a starburst activity but a nonthermal process in the central region is responsible for the radio emission from $1 \mathrm{H} 0323+342$.

\subsection{Doppler Factor}

If we assume the condition of energy equipartition between particles and magnetic fields, $T_{\mathrm{B} \text {,limit }} \sim 5 \times 10^{10} \mathrm{~K}$ is derived as a value for the upper limit in the source's rest frame (Readhead 1994). Observed $T_{\mathrm{B}, \text { rest }}^{\text {(image) }}$ for JVN epoch 1 significantly exceeds the limit. Thus the equipartition Doppler factor, $\delta_{\text {eq }}=T_{\mathrm{B}, \text { rest }}^{\text {(image) }} / T_{\mathrm{B} \text {,limit }}$, of greater than 1.7 should be required to reconcile with our $T_{\mathrm{B}}$. We can compute the variability Doppler factor as $\delta_{\mathrm{var}}=(1+z)\left(T_{\mathrm{B} \text {,rest }}^{\text {(var })} / T_{\mathrm{B}, \text { limit }}\right)^{1 /(3-\alpha)}$. Adopting $T_{\mathrm{B} \text {,rest }}^{(\mathrm{var})}=7.0 \times 10^{11} \mathrm{~K}$ and $\alpha_{8}^{15}=-0.70, \delta_{\mathrm{var}}=2.2$ can be obtained. Both $\delta_{\text {eq }}$ and $\delta_{\text {var }}$ are in good agreement with those obtained by the results with the F-GAMMA Program (Angelakis et al. 2012). These high $\delta$ indicate the existence of highly or mildly relativistic jet(s) in the inner region of the source. This is the third source in which the Dopplerbeaming effect has been detected in gamma-ray NLS1 by both direct imaging with VLBI and the flux variation, following PMN J0948+0022 (Doi et al. 2006b) and PKS 1502+036 (D’Ammando et al. 2013).

\subsection{Spectral Index Distribution}

Figure 4 shows the spectral index map of $1 \mathrm{H} 0323+342$ between JVN epoch 1 at $8.4 \mathrm{GHz}$ (2010 November 1) and the VLBA MOJAVE at $15.4 \mathrm{GHz}$ (2010 October 15). Both observations do not employ the phase-referencing technique, resulting in the loss of the absolute position through the self-calibration procedure (Pearson \& Readhead 1984; Thompson et al. 2001). We therefore superposed two images with reference to the optically-thin component D1 since the optically-thick central region generally shows the frequencydependent position shift (e.g., Lobanov 1998a b). Model fitting to the restored $15.4 \mathrm{GHz}$ MOJAVE image results in the position offset of the component D1 at 8.4 and $15.4 \mathrm{GHz}$ with respect to the component $\mathrm{C}$ to be $\Delta \alpha=0.2$ mas and $\Delta \delta=0.3$ mas in right ascension and declination, respectively. The compact central region with a size of 0.6 mas or $0.7 \mathrm{pc}$ shows an optically thick spectral feature while it becomes steeper along the stream of the jet. Figure 5 shows the spectral index distribution given by the position angle of 122.8 , corresponding to the direction of the component D1 with respect to $\mathrm{C}$ at JVN epoch 1. Error bars at each point in Figure 5are estimated by the image rms and the amplitude calibration error of each image. Maximum of the spectral index at the innermost 
region is $\alpha=0.42 \pm 0.20$, showing the central region to have an inverted or flat spectrum at even higher frequencies. This feature is similar to that of blazars, suggesting that the source has activities due to relativistic jets.

\subsection{Proper Motion of Each Component}

Figure 6 shows the distance of the components D2 and $\mathrm{D} 1(\mathrm{a}, \mathrm{b})$ from the core $(\mathrm{C}$ or $\mathrm{C} 0)$ as a function of the observed epoch. Although we cannot identify the component D1 with either D1a or D1b, if we adopt the identification of D1 with D1a the apparent proper motion of $-0.115 \pm 0.083 \mathrm{mas} \mathrm{yr}^{-1}$ is obtained by a weighted least-squares linear fit to the component $\mathrm{D} 1$, corresponding to the apparent velocity, $v_{\text {app }}$, of $(-0.45 \pm 0.32) c$. If we exclude the points for JVN epochs 2 and 3 for a linear fit to D1, the apparent proper motion of $0.031 \pm 0.011 \mathrm{mas} \mathrm{yr}^{-1}$ is obtained, corresponding to $v_{\text {app }}=(0.12 \pm 0.04) c$. For the component D2, the apparent

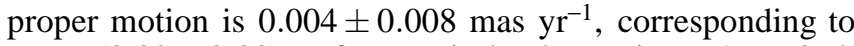
$v_{\text {app }}=(0.02 \pm 0.03) c$. If we omit the data point at 1996.374, the apparent proper motion becomes $0.033 \pm 0.004$ mas $_{\mathrm{yr}^{-1}}$, corresponding to $v_{\text {app }}=(0.13 \pm 0.02) c$, which is similar to that for the component D1. We cannot determine whether the component D1 at the epoch 1996.374 and at other epochs is identical since no VLBI observation was performed from 1996.374 to 2003.688. Future multiepoch and intensive VLBI observations will make an identification and a measurement of precise proper motion of D1. In either case, these show that both the components D2 and D1 are stationary, or have very low speeds compared to typical (gamma-ray) blazars (e.g., Jorstad et al. 2001).

\subsection{Relation to General Properties of NLS1s}

As shown in Section 11 NLS1s are generally considered to have higher accretion rates and lower jet activities compared to other types of radio-loud AGNs (Greene et al. 2006; Zhou et al. 2006). In the analogy of X-ray binaries with $M_{\mathrm{BH}} \sim 10 M_{\odot}$, radio emission is quenched when the luminosity is from a few \% to about $10 \%$ of the Eddington rate, corresponding to the high/soft state, while the jet is emerged when the luminosity is nearly equal to the Eddington rate, corresponding to very high state (Maccarone et al. 2003; Fender et al. 2004). 1H 0323+342 has both highly or mildly relativistic jets in the innermost region revealed with our observations and extremely high accretion rate with the accretion disk luminosity of $0.9 L_{\text {Edd }}$ (Abdo et al. 2009b). These imply that the state transition of the source is in accordance with that of Galactic X-ray binaries.

On the other hand, $1 \mathrm{H} 0323+342$ has very high accretion rate with a smaller black hole mass of $\sim 10^{7} M_{\odot}$ (Zhou et al. 2007; Abdo et al. 2009b), while it also shows very radio-loud feature of $R_{1.4}=318$ (Foschini 2011), or $R_{5}=246$ (Doi et al. 2012). These properties seem to be contrary to previous studies showing a correlation between radio loudness and the black hole mass (McLure \& Jarvis 2004, and references therein), and an anti-correlation between radio loudness and the accretion rate (Greene et al. 2006, and references therein) for a large sample of AGNs. Following these correlation, $1 \mathrm{H} 0323+342$ may be intrinsically a radio-quiet one but seem to be radio-loud as a result of the Doppler-boosting effect, as similar results have been obtained for several radio-loud NLS1s (Doi et al. 2011, 2012). Observed radio flux density is boosted by a factor of $D=\delta^{3-\alpha}$, and we obtain $D=18.5$ applying $\delta=2.2$ and $\alpha=-0.70$ derived from our studies. Thus the intrinsic radio loudness can be estimated as $R_{1.4}^{(\mathrm{int})}=17$ and $R_{5}^{\text {(int) }}=13$. Although these $R$ are still in the radio-loud regime $(R>10$; Kellermann et al. 1989), 1H 0323+342 intrinsically has much lower $R$ than expected.

\subsection{Radio Spectrum}

Figure 7 shows the composite radio spectrum of $1 \mathrm{H} 0323+342$, including not only our VLBI observation results but also previous single-dish or interferometric measurements. We applied spectral fitting to the VLBI data with a synchrotron self-absorption (SSA) spectral model, $S_{\nu}=$ $S_{0} \nu^{2.5}\left[1-\exp \left(-\tau_{\mathrm{ss}} \nu^{\alpha-2.5}\right)\right]$, where $S_{0}$ is a scaling constant and and $\tau_{\mathrm{ss}}$ is the SSA coefficient. In the SSA fit to the sum of the flux of all VLBI components, a peak flux density, $S_{\mathrm{m}}$, of $0.72 \mathrm{Jy}$ is derived at a turnover frequency, $\nu_{\mathrm{m}}$, of $3.5 \mathrm{GHz}$, as shown in Figure 7 (a), while the source is separated into two or more components in Figures 2 and 3 . If we apply the SSA fit to each VLBI component, as shown in Figure 7 (b), $S_{\mathrm{m}}=0.57 \mathrm{Jy}$ is derived at $\nu_{\mathrm{m}}=3.9 \mathrm{GHz}$ for the component C. $\nu_{\mathrm{m}}$ can be given under the condition of a homogeneous, self-absorbed, and incoherent synchrotron radio source with a power-law electron energy distribution as

$$
\nu_{\mathrm{m}} \sim 8 B^{1 / 5} S_{\mathrm{m}}^{2 / 5} \theta^{-4 / 5}(1+z)^{1 / 5} \quad[\mathrm{GHz}]
$$

(Kellermann \& Pauliny-Toth 1981), where $B$ [G] is the magnetic field, and $\theta$ [mas] is the angular size of a source. Applying $\nu_{\mathrm{m}}=3.9 \mathrm{GHz}$ and $S_{\mathrm{m}}=0.57 \mathrm{Jy}$ given by the SSA fitting results, and $\theta=\sqrt{\theta_{\text {maj }} \theta_{\min }} \sim 0.4$ mas from VLBI observations, we can derive $B \sim 2 \mathrm{mG}$, which is comparable to the magnetic field for young radio galaxies (e.g., Murgia et al. 1999). For the component D $1, S_{\mathrm{m}}=0.31 \mathrm{Jy}$ is derived assuming $\nu_{\mathrm{m}}=0.9 \mathrm{GHz}$ in the spectral fitting in Figure $7 \mathrm{~b}$ ). Applying $B \sim 2 \mathrm{mG}$ derived for the component $\mathrm{C}$, and $\theta \sim 2$ mas, we obtain $S_{\mathrm{m}} \sim 0.37$ Jy from Equation (3), which is comparable to that given by the SSA fitting. Although Equation (3) depends strongly on the parameters, the component D1 shows similar $\nu_{\mathrm{m}}$ or $S_{\mathrm{m}}$ between the spectral fitting and numerical estimation. In Figure 7 b), the sum of two synchrotron spectra accounts for the total flux measurements at a frequency of greater than $1 \mathrm{GHz}$. Especially, the total flux of the central component detected by VLA at $1.4 \mathrm{GHz}$ (open triangle in Figure 7, Doi et al. 2012) is accountable for the sum of the flux of two VLBI components, implying that the emission of the VLA central component mainly comes from the pc-scale VLBI components. However, the composite spectrum for the VLBI components cannot account for the points at MHz-frequency range. These emissions probably come from extended, optically-thin components on the kpc-scale detected with interferometric observations, as mentioned in Section 5.7

We should point out that the composite radio spectrum shown in Figure 7 is not obtained simultaneously. Future multifrequency, sumultaneous, and high-resolution VLBI observations are thus important to reveal spectral properties of the innermost region precisely.

\subsection{Possibility of Young Radio Source and Recurrent Jet Activity}

Some previous studies point out similarities between NLS1s and young radio galaxies, such as gigahertz-peaked spectrum sources (GPSs; O'Dea 1998), in terms of compact and stable radio morphologies and a steep spectrum of an inner region (e.g., Gallo et al. 2006; Komossa et al. 2006). As 
shown in Section 5.4, the JVN observations combined with the archival VLBA data revealed the existence of two stationary or slowly moving components D2 and D1, and Zhou et al. (2007) mention that the component D1 is possibly a weak radio lobe.

The viewing angle of the component, $\phi$, can be obtained using the apparent velocity, $\beta_{\text {app }} \equiv v_{\text {app }} / c$, and a Doppler factor, $\delta$, as

$$
\phi=\tan ^{-1} \frac{2 \beta_{\mathrm{app}}}{\beta_{\mathrm{app}}^{2}+\delta^{2}-1}
$$

(Ghisellini et al. 1993). If the component D1 has the same $\delta$ as $\mathrm{C}$ and we adopt $\delta=2.2$, as derived in Section 5.2 we can obtain $\phi=3.6$, which is in good agreement with $\phi=3^{\circ}$ derived by the model fitting to the spectral energy distribution (Abdo et al. 2009b). We can also calculate the real distance between $\mathrm{C}$ and $\mathrm{D} 1 \mathrm{as} 0.13 \mathrm{kpc}$. Assuming the linear expansion of the components D2 and D1 with the constant apparent velocity of $0.02 c$ and $0.12 c$, respectively, as shown in Section 5.4 the kinematic age, $t_{\text {kin }}$, of 120 and 220 years are derived for each component. Similar source age can be estimated from a correlation between the linear size and $\nu_{\mathrm{m}}$ of a source (O'Dea \& Baum 1997). Thus we believe that the pc-scale structure in $1 \mathrm{H} 0323+342$ detected by VLBI observations has GPS-like features in terms of the GHz-peaked spectrum as shown in Figure 7 the young age of $\sim 10^{2}$ years, and the compact structure of $\sim 0.1 \mathrm{kpc}$.

On the other hand, previous VLA observations show $t_{\text {kin }} \sim$ $10^{7}-10^{8}$ years for the $100 \mathrm{kpc}$-scale structure detected by the VLA C-array configuration (Antón et al. 2008; Doi et al. 2012), while $t_{\text {kin }} \sim 10^{6}-10^{7}$ years can be estimated for the extended structure with the scale of $20 \mathrm{kpc}$ detected by the VLA A-array configuration. The VLA images also show the extended structure with the position angle of $\sim 45^{\circ}$ with $100 \mathrm{kpc}$ scale and $\sim 90^{\circ}$ with $20 \mathrm{kpc}$ scale (Antón et al. 2008), both of which are significantly different with that of $\sim 125^{\circ}$ with pcscale images obtained by VLBI observations. Additionally, no extended feature could be found by the JVN observations within the field of view of a few hundred mas (corresponding to a kinematic age of $\sim 10^{4}$ years) under the condition of the detection limit as three times the rms image noise. These imply that the pc-scale jet structure represents recurrent jet activity (e.g., Baum et al. 1990; Augusto et al. 2006; Doi et al. $2013 \mathrm{~b})$. In fact, the $8 \mathrm{GHz}$ radio power for the pc-scale components is $P_{8 \mathrm{GHz}}=10^{24.6} \mathrm{~W} \mathrm{~Hz}^{-1}$, as shown in Section 4 and the jet kinetic power is roughly estimated to be $\sim 10^{44} \mathrm{erg} \mathrm{s}^{-1}$ from $P_{8 \mathrm{GHz}}$, which is enough to make the source grow to the size of a hundred kpc (Doi et al. 2012, 2013a). Extremely large difference in the position angle between kpc- and pcscales may be the projection effect of extended components due to the small viewing angle.

\subsection{Possibility of Free-Free Absorption}

Free-free absorption could be an explanation to make an apparent asymmetric structure, and previous VLBI observations have revealed the existence of free-free absorption in several GPSs (e.g., Kameno et al.|2000, 2001; Marr et al.|2001). The optical depth of free-free absorption can be estimated as

$$
\tau_{\mathrm{ff}}=8.235 \times 10^{-2} T_{\mathrm{e}}^{-1.35} \nu^{-2.1} \int_{\mathrm{LOS}} n_{\mathrm{e}}^{2} d l
$$

(Mezger \& Henderson 1967), where $T_{\mathrm{e}}[\mathrm{K}]$ and $n_{\mathrm{e}}\left[\mathrm{cm}^{-3}\right]$ are the electron temperature and density of the absorber, $\nu[\mathrm{GHz}]$ is the frequency, and $l[\mathrm{pc}]$ is the path length via the line of sight. Peak intensity of the component D1 is $38 \mathrm{mJy}_{\text {beam }}^{-1}$ on the image of JVN epoch 1, resulting in the attenuation of more than a factor of 4.1 if we assume the intrinsic jet symmetry and the detection limit of the counter component as three times the rms image noise. The $\tau_{\mathrm{ff}}$ of greater than 1.4 is thus required to reconcile with the observed asymmetry. Assuming $T_{\mathrm{e}}>8000 \mathrm{~K}$ for the fully ionized condition, $l=0.13 \mathrm{kpc}$ for the distance between the components $\mathrm{C}$ and $\mathrm{D} 1$, and $\nu=(1+z) \times 8.4=8.9 \mathrm{GHz}$, the column density of electrons $N_{\mathrm{e}}=n_{\mathrm{e}} l>6 \times 10^{23} \mathrm{~cm}^{-2}$ is required. This $N_{\mathrm{e}}$ is two orders of magnitude larger than the Galactic H I column density of $1.349 \times 10^{21} \mathrm{~cm}^{-2}$ obtained by the ROSAT All-Sky Survey (Voges et al. 1999). Moreover, the kpc-scale radio morphology of the source seems to be an asymmetric structure elongated to the east, as shown by Antón et al. (2008), although they consider the morphology to be a core plus a twosided structure. As shown in Section 5.5, we obtain $D=18.5$ as a boosting factor for the radio flux. This $D$ is accountable for the observed flux ratio of approaching to receding jet components mentioned above. We therefore conclude that the asymmetric structure detected by the JVN observations is not due to free-free absorption, but due to the Doppler-beaming effect.

\subsection{Gamma-Ray Emission from $1 \mathrm{H} 0323+342$}

Marscher et al. (2008) analyzed the multiwavelength light curve and quasi-simultaneous multi-epoch VLBI images of a blazar BL Lacertae, and proposed the inner jet model to explain the flare timing between each energy band and sudden change of the optical polarization position angle. They suggest that the flare is stimulated by the passage of the emission feature through the conical standing shock at around $10^{5} R_{\mathrm{s}}$ from the central black hole, where $R_{\mathrm{s}}$ is the Schwarzschild radius, and the gamma-ray flare would occur at this area. It corresponds to a linear scale of $0.1 \mathrm{pc}$ or an angular size of 0.08 mas for $1 \mathrm{H} 0323+342$. Although our observations combined with the archival VLBA results reveal the existence of the relativistic jet in the innermost region and stationary or slowly moving components D2 and D1, as shown in Section 5.4 the linear distance of D2 and D1 from C0 is $10^{7} R_{\mathrm{S}}$ and $10^{8} R_{\mathrm{s}}$, respectively, assuming $\phi$ from our results. The central black hole may be located upstream of the core at $8 \mathrm{GHz}$ as a result of the frequency-dependent position shift, thus the distance between the central black hole and the components D2 and D1 is farther than those mentioned above. On the other hand, the linear scale of $10^{5} R_{\mathrm{s}}$ is comparable to the light-crossing distance corresponding to the time scale of the short-term radio variability $\left(0.3 \times 10^{5} R_{\mathrm{s}}\right)$, which is probably associated with the component $\mathrm{C}$ (and the gamma-ray emitting region), as shown in Section 3.2. Moreover, the VLBA image at $15 \mathrm{GHz}$ shown in Figure 3 indicates the existence of a component D3 in the vicinity of the component $\mathrm{C} 0$ with a separation angle of 0.19 mas, or a linear distance of $2 \times 10^{5} R_{\mathrm{s}}$. Proper motion and flux variation of the component D3 might be related to the gamma-ray flux, and future high-resolution and high-sensitivity VLBI observations will be important to investigate the gamma-ray emission mechanism from NLS1s.

Gamma-ray emission has been detected from blazars with larger Doppler factor and smaller viewing angle (e.g., $\delta \gtrsim 14$ and $\phi<4$.8 for PKS 1741-038; Wajima et al. 2000), and with smaller Doppler factor and larger viewing angle (e.g., $\delta \sim 2.5$ and $\phi=23.3$ for PKS 1622-297; Wajima et al.2006). 
Both blazars have superluminal jet components and thus show very high intrinsic velocities with $\beta>0.9$, implying that the inverse-Compton process plays a key role for gamma-ray emission. On the other hand, $1 \mathrm{H} 0323+342$ has smaller $\delta$, $\beta$, and $\phi$ and thus seems to have different features compared to typical gamma-ray blazars. Future simultaneous, multiwavelength observations from radio to gamma-ray and highresolution polarization studies will be key issues to reveal the gamma-ray emission mechanism from NLS1s.

\section{CONCLUSION}

We made simultaneous single-dish and VLBI observations of a gamma-ray NLS1 galaxy $1 \mathrm{H} 0323+342$ at $8 \mathrm{GHz}$. The achievements of our study can be summarized as follows:

1. We found significant flux variation on the time scale of one month with the single-dish monitoring by Yamaguchi $32 \mathrm{~m}$ radio telescope. The total flux density varied by $5.5 \%$ in 32 days, corresponding to a variability brightness temperature of $7.0 \times 10^{11} \mathrm{~K}$.

2. Milliarcsecond-scale images obtained by three-epoch Japanese VLBI Network observations show that the source has a compact core-jet structure similar to that of blazars. The visibilities can be modeled satisfactorily by two elliptical Gaussian components. Only the central component shows flux decrease similar to that of the total flux obtained with the single-dish monitoring, while the flux density of the southeastern component seems to be stable.

3. Two-year Fermi/LAT monitoring results show that the source has gamma-ray flux variation on the similar time scale to our single-dish monitoring results. By combining the results 1 . and 2 ., we conclude that the source of short-term radio variability is probably associated with the gamma-ray emitting region.

4. The brightness temperature obtained by the JVN observations is greater than $(5.2 \pm 0.3) \times 10^{10} \mathrm{~K}$, and the radio power at $8 \mathrm{GHz}$ is estimated to be $10^{24.6} \mathrm{~W} \mathrm{~Hz}^{-1}$. These indicate that a nonthermal process in the central region is responsible for the radio emission from the source.

5. The Doppler factor is estimated to be $\delta_{\text {eq }}>1.7$ assuming the condition of energy equipartition in the JVN observations, and $\delta_{\mathrm{var}}=2.2$ from the observed radio variability, indicating the existence of relativistic jet(s). This is the third source in which the Doppler beaming effect is detected in gamma-ray NLS1s by both direct imaging with VLBI and the flux variation.

6. The JVN observations revealed the existence of Doppler-boosted jet components which affect radio loudness of the source. Although the source shows extremely high apparent radio loudness with $R>200$, the intrinsic $R$ is less than 20 applying the Doppler factor obtained by the JVN observations. Hence, for $1 \mathrm{H} 0323+342$ with a smaller black hole mass $(\sim$ $\left.10^{7} M_{\odot}\right)$ and high accretion rate $\left(\sim 0.9 L_{\text {Edd }}\right)$, there seems to be no large discrepancy with previous studies suggesting a correlation between $R$ and the black hole mass, and anti-correlation between $R$ and the accretion rate.
7. Multiepoch JVN and VLBA images detected slowly moving jet components D2 and D1 with apparent velocities of $(0.02 \pm 0.03) c$ and $(0.12 \pm 0.04) c$, respectively, and the real distance between the components $\mathrm{C}$ and D1 was estimated as $0.13 \mathrm{kpc}$. The kinematic age of $\sim 10^{2}$ years is derived for both components assuming the linear expansion, which is significantly different with that of kpc-scale components $\left(\sim 10^{7}-10^{8}\right.$ years $)$. By combining the results of the spectral fitting to the flux measurements and observed properties in terms of the position angle and lack of medium-scale radio emission, we conclude that the pc-scale jet structure represents recurrent jet activity.

We are grateful to the anonymous referee for valuable comments which improved the manuscript. The JVN project is led by the National Astronomical Observatory of Japan (NAOJ), which is a branch of the National Institutes of Natural Sciences (NINS), Hokkaido University, Ibaraki University, University of Tsukuba, Gifu University, Osaka Prefecture University, Yamaguchi University, and Kagoshima University, in cooperation with the Geographical Survey Institute (GSI), the Japan Aerospace Exploration Agency (JAXA), and the National Institute of Information and Communications Technology (NICT). The VLBA is operated by the National Radio Astronomy Observatory, which is a facility of the National Science Foundation operated under cooperative agreement by Associated Universities, Inc. This research has made use of the following data, tools, and facilities; the data from the MOJAVE database that is maintained by the MOJAVE team (Lister et al. 2009), the Swinburne University of Technology software correlator (Deller et al. 2011), NASA's Astrophysics Data System Abstract Service, the NASA/IPAC Extragalactic Database (NED), which is operated by the Jet Propulsion Laboratory, Ned Wright's on-line cosmology calculator, and the VizieR catalogue access tool, CDS, Strasbourg, France (Ochsenbein et al. 2000). This work is partly supported by the National Natural Science Foundation of China (grant 11121062), the CAS/SAFEA International Partnership Program for Creative Research Teams, and the Strategic Priority Research Program on Space Science, the Chinese Academy of Sciences (Grant No. XDA04060700).

\section{REFERENCES}

Abdo, A. A., Ackermann, M., Ajello, M., et al. 2009a, ApJ, 699, 976 Abdo, A. A., Ackermann, M., Ajello, M., et al. 2009b, ApJ, 707, L142 Angelakis, E., Fuhrmann, L., Nestoras, I., et al. 2012, arXiv:1205.1961 Antón, S., Browne, I. W. A., \& Marchã, M. J. 2008, A\&A, 490, 583 Augusto, P., Gonzalez-Serrano, J. I., Perez-Fournon, I., \& Wilkinson, P. N. 2006, MNRAS, 368, 1411

Beasley, A. J., Gordon, D., Peck, A. B., et al. 2002, ApJS, 141, 13

Baum, S. A., O’Dea, C. P., Murphy, D. W., \& de Bruyn, A. G. 1990, A\&A, 232, 19

Boroson, T. A. 2002, ApJ, 565, 78

Calderone, G., Foschini, L., Ghisellini, G., et al. 2011, MNRAS, 413, 2365

Colla, G., Fanti, C., Fanti, R., et al. 1973, A\&AS, 11, 291

Condon, J. J., Cotton, W. D., Greisen, E. W., et al. 1998, AJ, 115, 1693

D'Ammando, F., Orienti, M., Doi, A., et al. 2013, MNRAS, 433, 952

D'Ammando, F., Orienti, M., Finke, J., et al. 2012, MNRAS, 426, 317

Deller, A. T., Brisken, W. F., Phillips, C. J., et al. 2011, PASP, 123, 275

Doi, A., Asada, K., Fujisawa, K., et al. 2013a, ApJ, 765, 69

Doi, A., Asada, K., \& Nagai, H. 2011, ApJ, 738, 126

Doi, A., Fujisawa, K., Inoue, M., et al. 2007, PASJ, 59, 703

Doi, A., Fujisawa, K., Harada, K., et al. 2006a, in Proc. 8th European VLBI

Network Symp., ed. W. Baan, R. Bachiller, R. Booth et al. (Bonn:

MPIfR), 71 
Doi, A., Murata, Y., Mochizuki, N., et al. 2013b, PASJ, 65, 57

Doi, A., Nagai, H., Asada, K., et al. 2006, PASJ, 58, 829

Doi, A., Nagira, H., Kawakatu, N., et al. 2012, ApJ, 760, 41

Douglas, J. N., Bash, F. N., Bozyan, F. A., Torrence G. W., \& Wolfe, C. 1996, AJ, 111, 1945

Fender, R. P., Belloni, T. M., \& Gallo, E. 2004, MNRAS, 355, 1105

Fomalont, E. B. 1999, in ASP Conf. Ser. 180, Synthesis Imaging in Radio Astronomy II, ed. G. B. Taylor, C. L. Carilli, \& R. A. Perley (San Francisco: ASP), 301

Foschini, L. 2011, in Narrow-Line Seyfert 1 Galaxies and Their Place in the Universe, ed. L. Foschini, M. Colpi, L. Gallo et al. (Trieste: PoS), 024

Fuhrmann, L, Angelakis, E., Nestoras, I., et al. 2011, in Narrow-Line Seyfert 1 Galaxies and Their Place in the Universe, ed. L. Foschini, M. Colpi, L. Gallo et al. (Trieste: PoS), 026

Fujisawa, K., Mashiyama, H., Shimoikura, T., \& Kawaguchi, N. 2002, in Proc. IAU 8th Asian-Pacific Regional Meeting, Vol. 2, ed. S. Ikeuchi, J. Hearnshaw, \& T. Hanawa (Tokyo: Astron. Soc. Japan), 3

Gallo, L. C., Edwards, P. G., Ferrero, E., et al. 2006, MNRAS, 370, 245

Ghisellini, G., Padovani, P., Celotti, A., \& Maraschi, L. 1993, ApJ, 407, 65

Giroletti, M., Paragi, Z., Bignall, H., et al. 2011, A\&A, 528, L11

Greene, J. E., Ho, L. C., \& Ulvestad, J. S. 2006, ApJ, 636, 56

Greisen, E. W. 2003, in Information Handling in Astronomy - Historical Vistas, ed. A. Heck (Dordrecht: Kluwer), 109

Hartman, R. C., Bertsch, D. L., Bloom, S. D., et al. 1999, ApJS, 123, 79

Healey, S. E., Romani, R. W., Taylor, G. B., et al. 2007, ApJS, 171, 61

Jorstad, S. G., Marscher, A. P., Mattox, J. R., et al. 2001, ApJS, 134, 181

Kadota, A., Fujisawa, K., Sawada-Satoh, S., Wajima, K., \& Doi, A. 2012, PASJ, 64, 109

Kameno, S., Horiuchi, S., Shen, Z.-Q, et al. 2000, PASJ, 52, 209

Kameno, S., Sawada-Satoh, S., Inoue, M., Shen, Z.-Q., \& Wajima, K. 2001, PASJ, 53, 169

Kellermann, K. I., \& Pauliny-Toth, I. I. K. 1981, ARA\&A, 19, 373

Kellermann, K. I., Sramek, R., Schmidt, M., Shaffer, D. B., \& Green, R. 1989, AJ, 98, 1195

Kobayashi, H., Sasao, T., Kawaguchi, N., et al. 2003, in ASP Conf. Ser. 306, New Technologies in VLBI, ed. Y. C. Minh (San Francisco, CA: ASP), 48

Komatsu, E., Dunkley, J., Nolta, M. R., et al. 2009, ApJS, 180, 330

Komossa, S., Voges, W., Xu, D., et al. 2006, AJ, 132, 531

Linford, J. D., Taylor, G. B., Romani, R. W., et al. 2012, ApJ, 744, 177

Lister, M. L., Aller, H. D., Aller, M. F., et al. 2009, AJ, 137, 3718

Lobanov, A. P. 1998a, A\&A, 330, 79

Lobanov, A. P. 1998b, A\&AS, 132, 261

Lonsdale, C. J., Smith, H. E., \& Lonsdale, C. J. 1993, ApJ, 405, L9

Maccarone, T. J., Gallo, E., \& Fender, R. 2003, MNRAS, 345, L19

Marchã, M. J. M., Browne, I. W. A., Impey, C. D., \& Smith, P. S. 1996, MNRAS, 281, 425

Marr, J. M., Taylor, G. B., \& Crawford, F., III 2001, ApJ, 550, 160

Marscher, A. P., Jorstad, S. G., D'Arcangelo, F. D., et al. 2008, Nature, 452, 966
McLure, R. J., \& Jarvis, M. J. 2004, MNRAS, 353, L45

Mezger, P. G., \& Henderson, A. P. 1967, ApJ, 147, 471

Mineshige, S., Kawaguchi, T., Takeuchi, M., \& Hayashida, K. 2000, PASJ, 52,499

Murgia, M., Fanti, C., Fanti, R., et al. 1999, A\&A, 345, 769

Neumann, M., Reich, W., Fürst, E., et al. 1994, A\&AS, 106, 303

Nolan, P. L., Abdo, A. A., Ackermann, M., et al. 2012, ApJS, 199, 31

Ochsenbein, F., Bauer, P., \& Marcout, J. 2000, A\&AS, 143, 23

O'Dea, C. P., \& Baum, S. A. 1997, AJ, 113, 148

O’Dea, C. P. 1998, PASP, 110, 493

Orienti, M., D'Ammando, F., \& Giroletti, M., for the Fermi-LAT

Collaboration 2012, arXiv: 1205.0402

Osterbrock, D. E., \& Pogge, R. W. 1985, ApJ, 297, 166

Ott, M., Witzel, A., Quirrenbach, A., et al. 1994, A\&A, 284, 331

Pearson, T. J., \& Readhead, A. C. S. 1984, ARA\&A, 22, 97

Pogge, R. W. 2000, New Astron. Rev., 44, 381

Readhead, A. C. S. 1994, ApJ, 426, 51

Reich, W., Fürst, E., Reich, P., et al. 2000, A\&A, 363, 141

Rengelink, R. B., Tang, Y., de Bruyn, A. G., et al. 1997, A\&AS, 124, 259

Shepherd, M. C. 1997, in ASP Conf. Ser. 125, Astronomical Data Analysis Software and Systems VI, ed. G. Hunt \& H. E. Payne (San Francisco: ASP), 77

Shibata, K. M., Kameno, S., Inoue, M., \& Kobayashi, H. 1998, in ASP Conf. Ser. 144, IAU Colloq. 164: Radio Emission from Galactic and Extragalactic Compact Sources, ed. J. A. Zensus, G. B. Taylor, \& J. M. Wrobel (San Francisco: ASP), 413

Smith, D. A., Herter, T., \& Haynes, M. P. 1998a, ApJ, 494, 150

Smith, H. E., Lonsdale, C. J., \& Lonsdale, C. J. 1998b, ApJ, 492, 137

Sowards-Emmerd, D., Romani, R. W., \& Michelson, P. F. 2003, ApJ, 590, 109

Sowards-Emmerd, D., Romani, R. W., Michelson, P. F., \& Ulvestad, J. S. 2004, ApJ, 609, 564

Thompson, A. R., Moran, J. M., \& Swenson, G. W., Jr. 2001, Interferometry and Synthesis in Radio Astronomy, 2nd ed. (New York: John Wiley \& Sons)

Voges, W., Aschenbach, B., Boller, Th., et al. 1999, A\&A, 349, 389

Vollmer, B., Gassmann, B., Derrière, S., et al. 2010, A\&A, 511, 53

Wagner, S. J., \& Witzel, A. 1995, ARA\&A, 33,163

Wajima, K., Bignall, H. E., Kobayashi, H., et al. 2006, PASJ, 58, 223

Wajima, K., Lovell, J. E. J., Kobayashi, H., et al. 2000, PASJ, 52, 329

Yonekura, Y., Saito, Y., Saito, T., et al. 2013, in ASP Conf. Ser. 476, New

Trends in Radio Astronomy in the ALMA Era, ed. R. Kawabe \& N. Kuno (San Francisco, CA: ASP), 415

Yuan, W., Zhou, H. Y., Komossa, S., et al. 2008, ApJ, 685, 801

Zhang, X., Zheng, Y., Chen, H., et al. 1997, A\&AS, 121, 59

Zhou, H.-Y., Wang, T.-G., Dong, X.-B., Zhou, Y.-Y., \& Li, C. 2003, ApJ, 584,147

Zhou, H.-Y., Wang, T.-G., Yuan, W.-M., et al. 2006 ApJS, 166, 128

Zhou, H.-Y., Wang, T.-G., Yuan, W.-M., et al. 2007, ApJ, 658, L13 


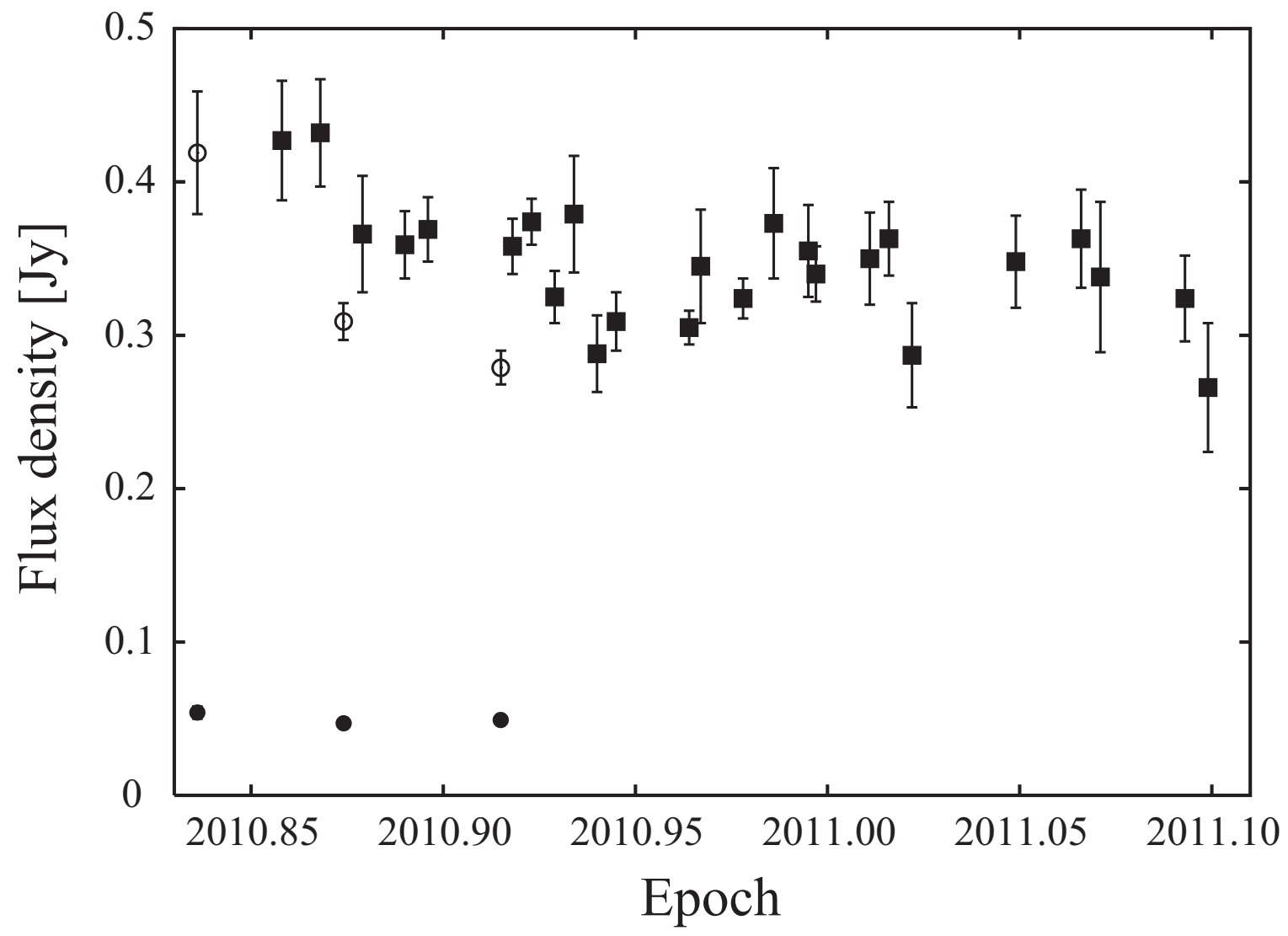

Figure 1. $8.4 \mathrm{GHz}$ light curve of $1 \mathrm{H} 0323+342$. The filled squares show the total flux obtained with Yamaguchi $32 \mathrm{~m}$ radio telescope. Numerical data of the measurements are shown in Table 2 The open and filled circles indicate the flux of the components C and D1, respectively, obtained with the JVN observations (see also Section 3.2 and Table 1 . 


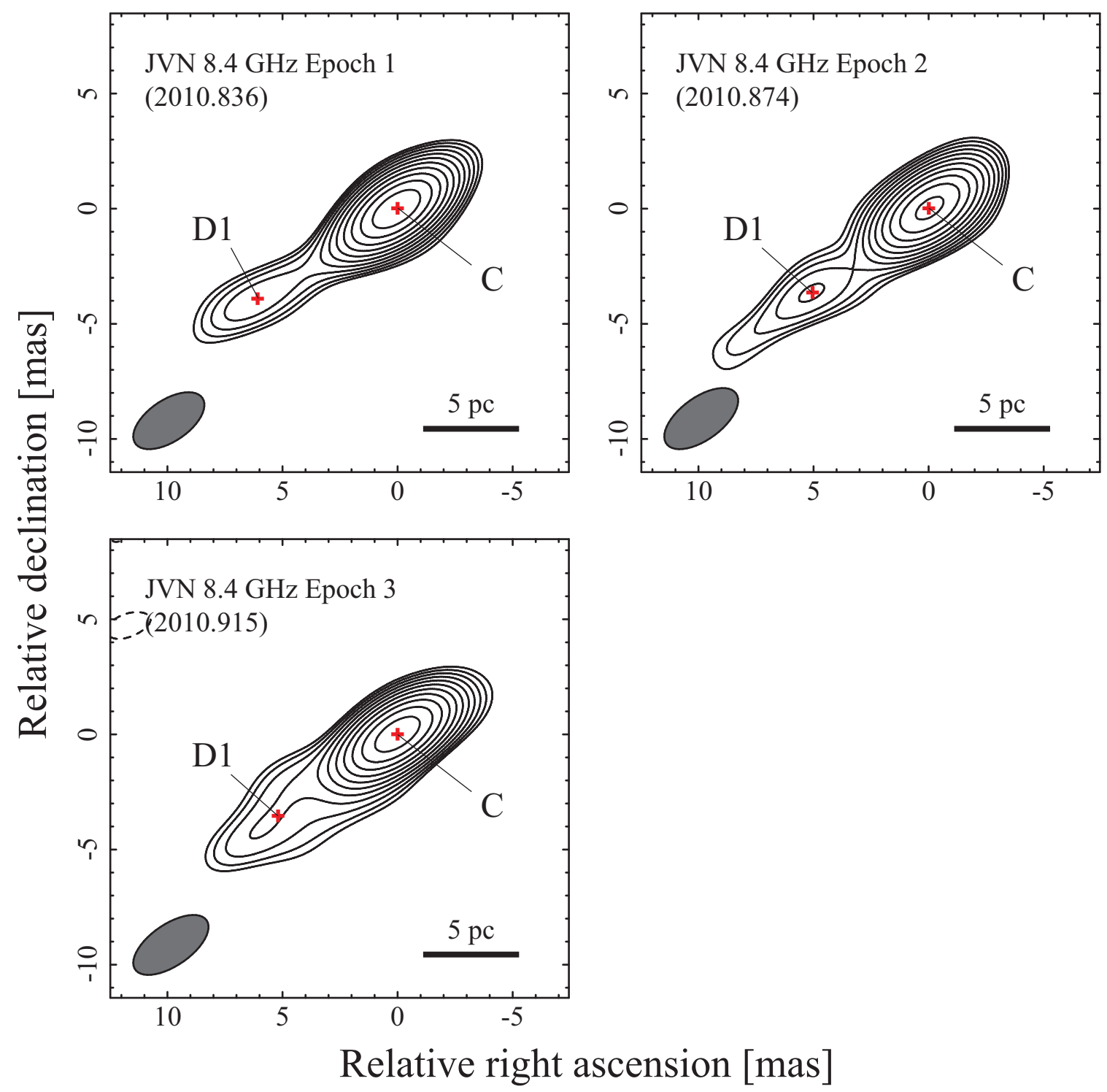

Figure 2. VLBI images of $1 \mathrm{H} 0323+342$ at epochs 1 (top left), 2 (top right), and 3 (bottom) obtained by the JVN observations at $8.4 \mathrm{GHz}$. The lowest contour is 3 times the off-source rms noise $(\sigma)$. The contour levels are $-3 \sigma, 3 \sigma \times(\sqrt{2})^{n}(n=0,1,2, \cdots, 10)$. Dashed and solid curves show negative and positive contours, respectively. The restoring beam is indicated at the lower left corner of each image. The labels C and D1 show the Gaussian model fitting components and the position of each component is indicated by the cross. The image descriptions are shown in Table 1 

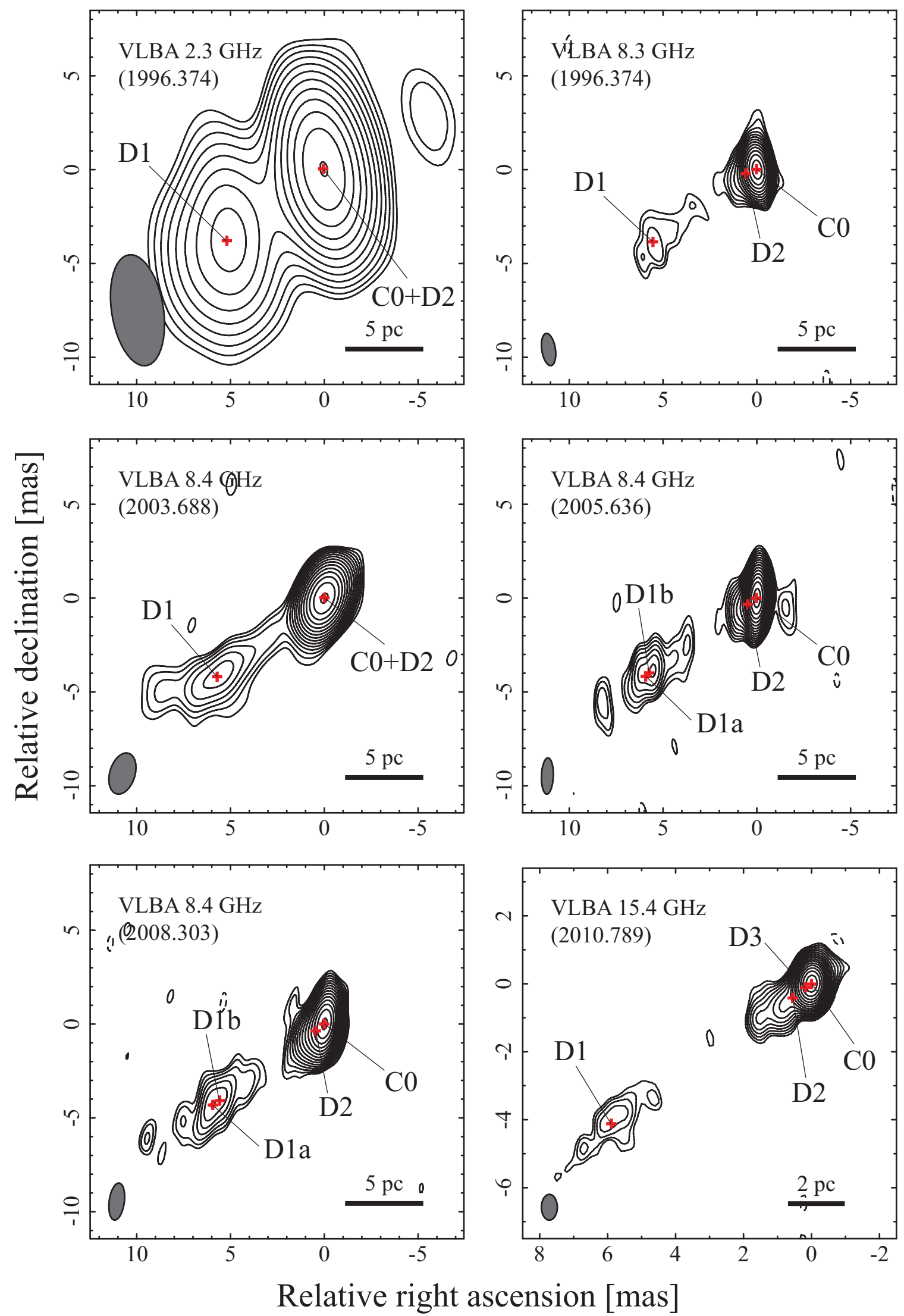

Figure 3. VLBI images of $1 \mathrm{H} 0323+342$ obtained by the VLBA archive data. Top left and bottom right panels show images at $2.3 \mathrm{GHz}$ and $15.4 \mathrm{GHz}$, respectively, while others are at $8 \mathrm{GHz}$. The lowest contour is 3 times the off-source rms noise $(\sigma)$. Dashed and solid curves show negative and positive contours, respectively. The restoring beam is indicated at the lower left corner of each image. The labels C0, D3, D2, and D1(a, b) show the Gaussian model fitting components and the position of each component is indicated by the cross. The image descriptions are shown in Table 4 


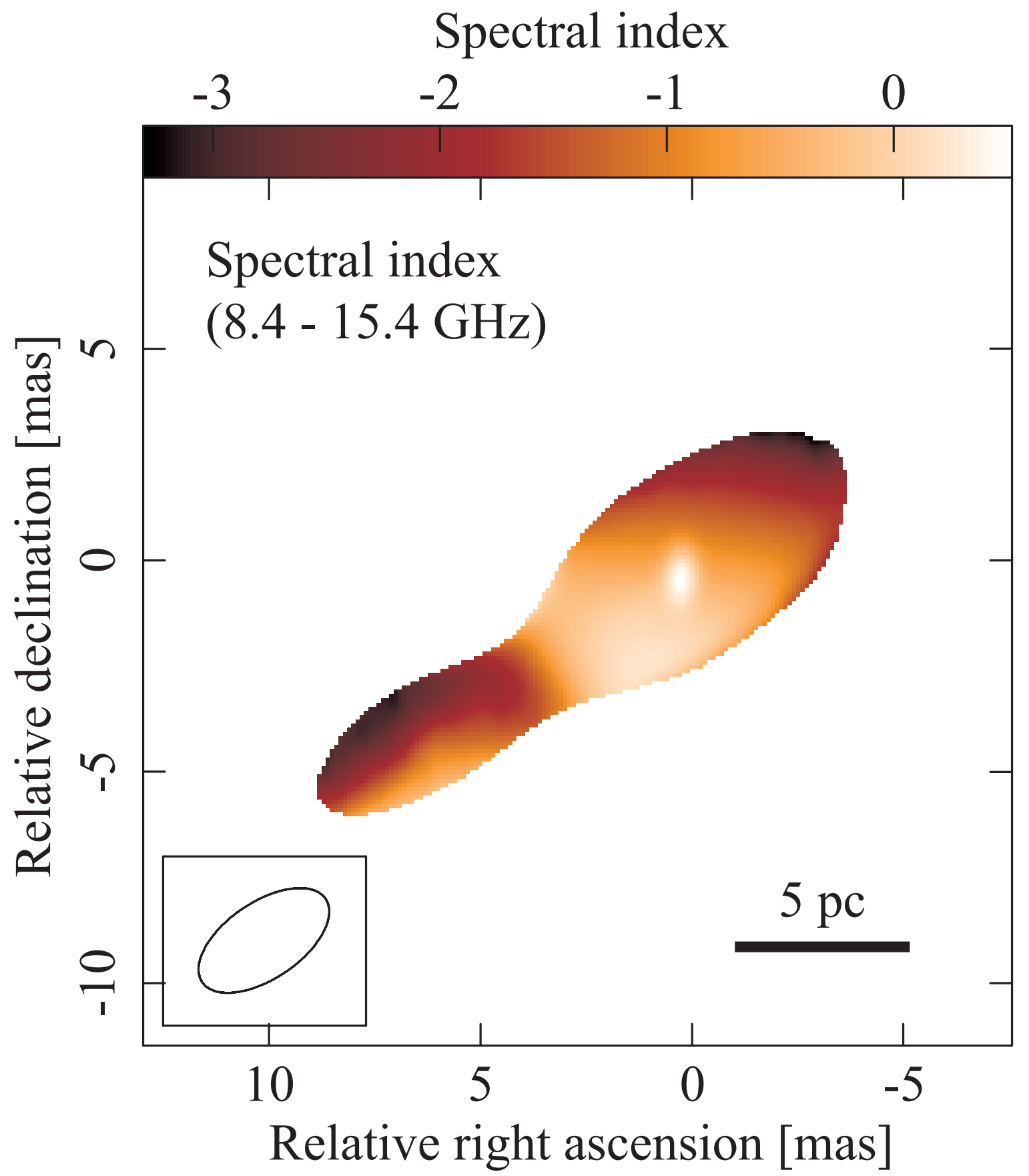

Figure 4. Spectral index map of $1 \mathrm{H} 0323+342$ derived from the flux densities at $8.4 \mathrm{GHz}$ (JVN epoch1 on 2010 November 1) and 15.4 GHz (VLBA MOJAVE on 2010 October 15). The map corresponds to the area greater than $3 \sigma$ noise level in JVN epoch 1 . The $15.4 \mathrm{GHz}$ map is restored with the same beam size as the $8.4 \mathrm{GHz}$ map, which is represented in the lower left corner 


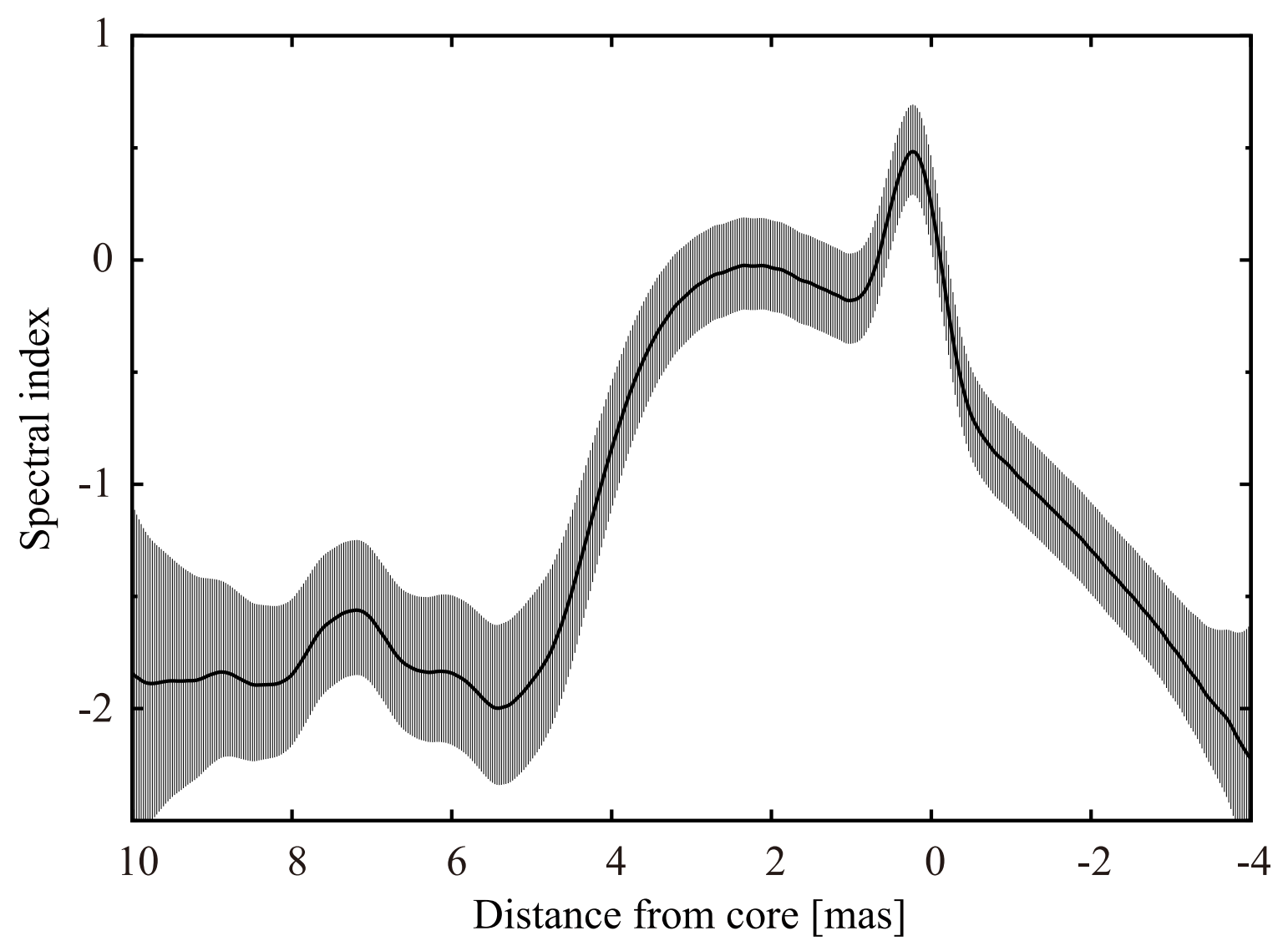

Figure 5. Spectral index distribution given by the position angle of 122.8 . The horizontal axis shows the angular separation from the center position of the component $\mathrm{C}$. A positive value corresponds to the southeastern direction. 


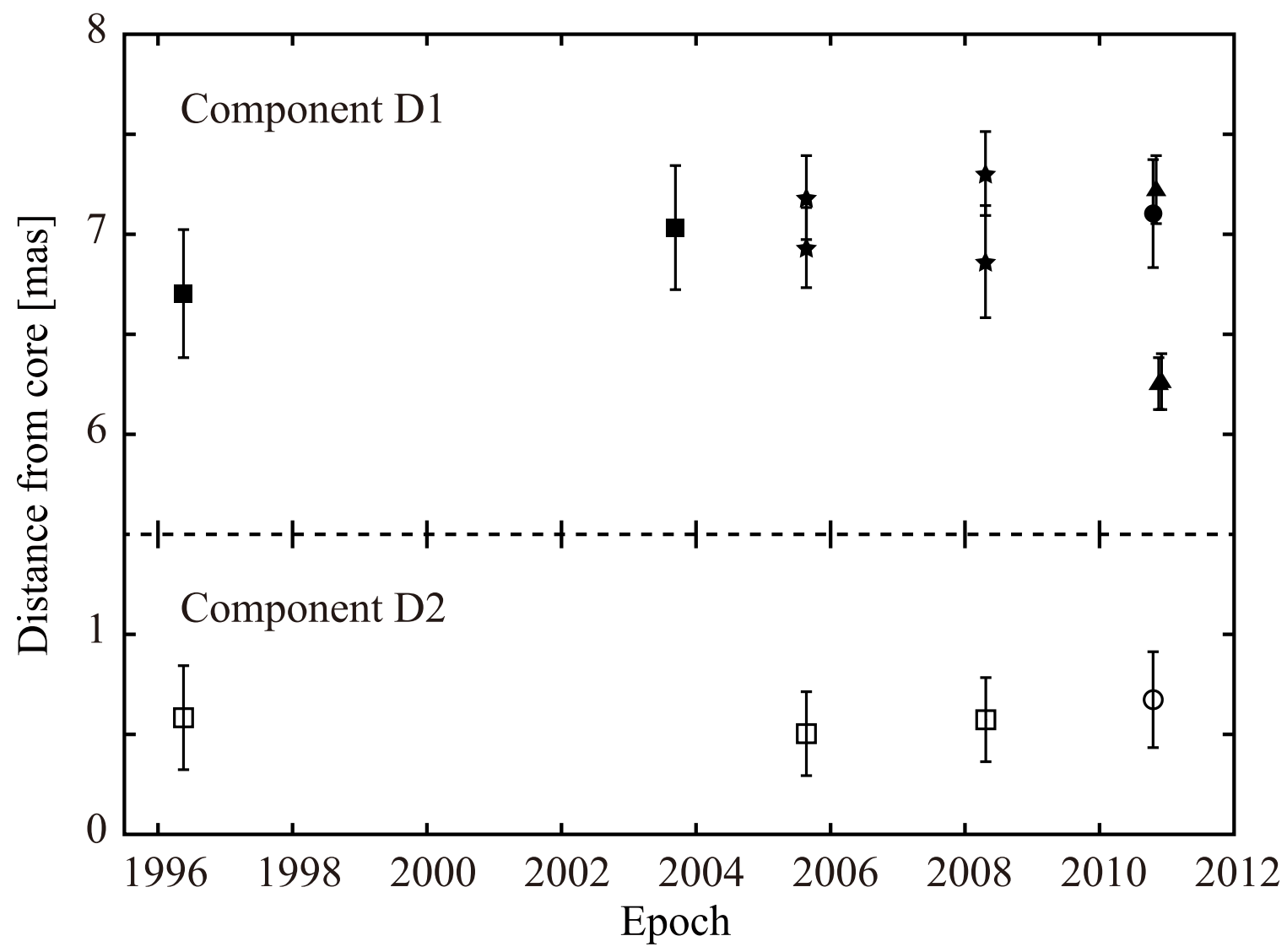

Figure 6. Distance of the components D2 and D1(a, b) from the component C (for JVN observations) or C0 (for VLBA observations) as a function of the observed epoch. See Tables 3 and 5 for numerical values. The open and filled symbols are for the components D2 and D1, respectively. Squares and circles represent the VLBA results at 8 and $15 \mathrm{GHz}$, respectively, while stars correspond to the components D1a and D1b at epoch 2005.636 and 2008.303. Triangles represent the JVN results.

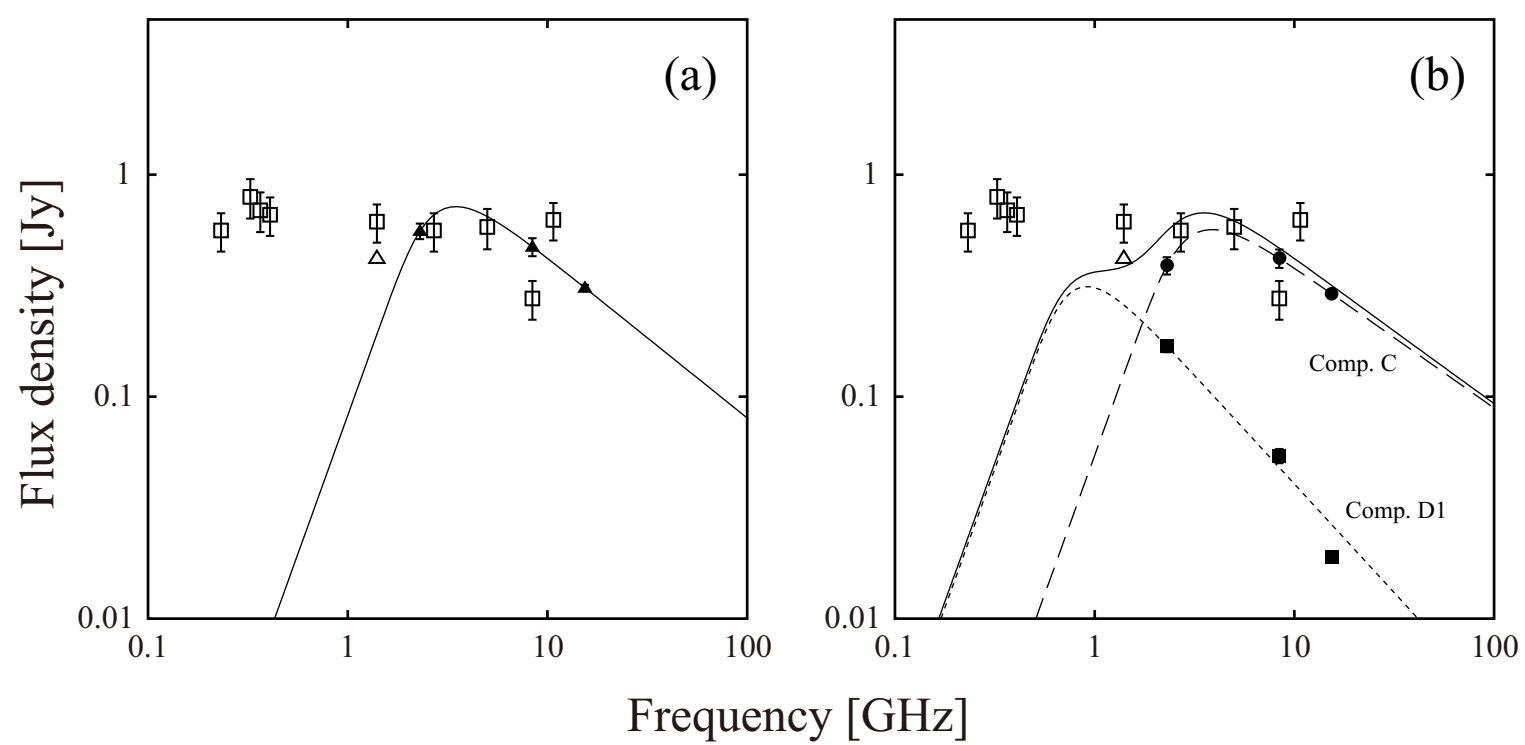

Figure 7. Composite radio spectrum of $1 \mathrm{H} 0323+342$. The filled symbols represent the VLBI results at $2.3 \mathrm{GHz}$ (VLBA), $8.4 \mathrm{GHz}$ (JVN epoch 1 ), and $15.4 \mathrm{GHz}$ (VLBA), while the open squares are the total flux density by single-dish or radio interferometer. The open triangle indicates the flux density of the core component obtained by VLA (Doi et al. 2012). (a) Radio spectrum for the sum of flux for all VLBI components. The solid line represents the best-fit curve with synchrotron spectrum. (b) Radio spectra for the components C and D1. The filled circles and squares are VLBI results for the components C (or C0 +D3 +D2 for VLBA) and D1, respectively. The dashed and dotted lines represent the best-fit curve with synchrotron spectrum for the components C and D1, respectively, and the solid line describes the sum of two synchrotron spectra. The turnover frequency of $0.9 \mathrm{GHz}$ is assumed for the spectral fitting to the component D1 (see also Section 5.7).

Single-dish and interferometer measurement data are from Colla et al. (1973), Condon et al. (1998), Douglas et al. (1996), Healey et al. (2007), Neumann et al. (1994), Reich et al. (2000), Rengelink et al. (1997), and Zhang et al. (1997). A part of the flux data is compiled from the SPECFIND V2.0 catalog (Vollmer et al. 2010). 
Table 1

Summary of JVN observations and description of JVN images.

\begin{tabular}{|c|c|c|c|c|c|c|c|c|}
\hline $\begin{array}{l}\text { Epoch } \\
\text { (1) }\end{array}$ & $\begin{array}{c}\text { Date (UT) } \\
\text { (2) }\end{array}$ & $\begin{array}{c}\text { Telescopes } \\
\text { (3) }\end{array}$ & $\begin{array}{c}\theta_{\text {Bmaj }} \\
{[\mathrm{mas}]} \\
(4)\end{array}$ & $\begin{array}{c}\theta_{\mathrm{Bmin}} \\
{[\mathrm{mas}]} \\
(5)\end{array}$ & $\begin{array}{l}\text { PA } \\
\text { [deg] } \\
(6)\end{array}$ & $\begin{array}{c}S_{\text {peak }} \\
{\left[\mathrm{mJy} \mathrm{beam}^{-1} \text { ] }\right.} \\
(7)\end{array}$ & $\begin{array}{c}\sigma \\
{\left[\begin{array}{c}\mathrm{mJy}_{\text {beam }}^{-1} \\
(8)\end{array}\right.} \\
\end{array}$ & $\begin{array}{c}S_{\text {CLEAN }} \\
{[\text { mJy] }} \\
(9)\end{array}$ \\
\hline 1 & 2010 Nov $111: 00-21: 00$ & VERA, HIT & 3.52 & 1.81 & 124.0 & 397 & 3.1 & 467 \\
\hline 2 & 2010 Nov 15 10:00 - 20:00 & VERA, HIT, KAS & 3.73 & 1.85 & 126.0 & 298 & 2.8 & 359 \\
\hline 3 & 2010 Nov 30 09:00 - 19:00 & VERA, HIT, KAS & 3.77 & 1.80 & 124.5 & 272 & 2.2 & 335 \\
\hline
\end{tabular}

Note. - Column 1: JVN observation epoch; Column 2: observation date; Column 3: telescopes: VERA - VERA $(4 \times 20 \mathrm{~m})$, HIT - Hitachi 32 m, KAS - Kashima $34 \mathrm{~m}$; Columns 4, 5, and 6: parameters of the restoring beam: Full-width at half maximum (FWHM) of major and minor axes and the position angle of the major axis; Column 7: peak intensity; Column 8: rms noise level; Column 9: total CLEANed flux.

Table 2

Results of the total flux measurement at $8.38 \mathrm{GHz}$ with Yamaguchi $32 \mathrm{~m}$ radio telescope.

\begin{tabular}{lccc}
\hline \hline \multicolumn{1}{c}{ Date } & Epoch & $\begin{array}{c}S_{8 \mathrm{GHz}} \\
{[\mathrm{mJy}]}\end{array}$ & $N^{\mathrm{a}}$ \\
\hline 2010 Nov 9 & 2010.858 & $427 \pm 28$ & 31 \\
2010 Nov 13 & 2010.868 & $432 \pm 24$ & 35 \\
2010 Nov 17 & 2010.879 & $366 \pm 27$ & 32 \\
2010 Nov 21 & 2010.890 & $359 \pm 16$ & 39 \\
2010 Nov 23 & 2010.896 & $369 \pm 15$ & 38 \\
2010 Dec 1 & 2010.918 & $358 \pm 18$ & 39 \\
2010 Dec 3 & 2010.923 & $374 \pm 11$ & 39 \\
2010 Dec 5 & 2010.929 & $325 \pm 12$ & 39 \\
2010 Dec 7 & 2010.934 & $379 \pm 27$ & 38 \\
2010 Dec 9 & 2010.940 & $288 \pm 18$ & 38 \\
2010 Dec 11 & 2010.945 & $309 \pm 13$ & 40 \\
2010 Dec 18 & 2010.964 & $305 \pm 8$ & 39 \\
2010 Dec 19 & 2010.967 & $345 \pm 26$ & 26 \\
2010 Dec 23 & 2010.978 & $324 \pm 9$ & 36 \\
2010 Dec 26 & 2010.986 & $373 \pm 25$ & 24 \\
2010 Dec 29 & 2010.995 & $355 \pm 21$ & 31 \\
2010 Dec 30 & 2010.997 & $340 \pm 13$ & 39 \\
2011 Jan 4 & 2011.011 & $350 \pm 21$ & 26 \\
2011 Jan 6 & 2011.016 & $363 \pm 17$ & 36 \\
2011 Jan 8 & 2011.022 & $287 \pm 24$ & 25 \\
2011 Jan 18 & 2011.049 & $348 \pm 21$ & 17 \\
2011 Jan 24 & 2011.066 & $363 \pm 23$ & 18 \\
2011 Jan 26 & 2011.071 & $338 \pm 35$ & 12 \\
2011 Feb 3 & 2011.093 & $324 \pm 20$ & 15 \\
2011 Feb 5 & 2011.099 & $266 \pm 30$ & 12 \\
\hline
\end{tabular}

${ }^{a}$ Number of independent measurements.

Table 3

Model fitting results for JVN observations.

\begin{tabular}{|c|c|c|c|c|c|c|c|c|}
\hline Epoch & Comp. & $\begin{array}{c}S \\
{\left[\begin{array}{c}\mathrm{mJy}] \\
(3)\end{array}\right.}\end{array}$ & $\begin{array}{c}r \\
{[\mathrm{mas}]} \\
(4)\end{array}$ & $\begin{array}{c}\phi \\
{[\mathrm{deg}]} \\
(5)\end{array}$ & $\begin{array}{c}\theta_{\mathrm{maj}} \\
{[\mathrm{mas}]} \\
(6)\end{array}$ & $\begin{array}{c}\theta_{\min } \\
{[\mathrm{mas}]} \\
(7)\end{array}$ & $\begin{array}{c}\text { PA } \\
\text { [deg] } \\
(8)\end{array}$ & $\begin{array}{c}T_{\mathrm{B}, \text { rest }}^{\text {(image) }} \\
{\left[10^{10} \mathrm{~K}\right]} \\
(9)\end{array}$ \\
\hline 1 & $\begin{array}{l}\mathrm{C} \\
\mathrm{D} 1\end{array}$ & $\begin{array}{r}419 \pm 40 \\
54 \pm 4\end{array}$ & $\begin{array}{c}\cdots \\
7.22 \pm 0.17\end{array}$ & $\begin{array}{c}\ldots \\
122.8 \pm 2.1\end{array}$ & $\begin{array}{r}<1.12 \pm 0.11 \\
3.76 \pm 0.38\end{array}$ & $\begin{array}{r}<0.12 \pm 0.01 \\
0.11 \pm 0.01\end{array}$ & $\begin{array}{l}131.1 \pm 0.4 \\
116.5 \pm 2.7\end{array}$ & $>8.3 \pm 1.3$ \\
\hline 2 & $\begin{array}{c}\mathrm{C} \\
\mathrm{D} 1\end{array}$ & $\begin{array}{r}309 \pm 12 \\
47 \pm 1\end{array}$ & $6.25+0.13$ & $\begin{array}{c}\cdots \\
125.8+19\end{array}$ & $\begin{array}{r}<0.59 \pm 0.02 \\
2.76 \pm 0.11\end{array}$ & $\begin{array}{r}<0.24 \pm 0.01 \\
0.27 \pm 0.01\end{array}$ & $\begin{array}{r}84.6 \pm 0.5 \\
109.6 \pm 3.4\end{array}$ & $\begin{array}{c}>5.8 \pm 0.4 \\
\ldots\end{array}$ \\
\hline 3 & $\begin{array}{l}\mathrm{C} \\
\mathrm{D} 1\end{array}$ & $\begin{array}{r}279 \pm 11 \\
49 \pm 1\end{array}$ & $\begin{array}{c}\ldots \\
6.26 \pm 0.14\end{array}$ & $\begin{array}{c}\cdots \\
124.2 \pm 1.4\end{array}$ & $\begin{array}{r}<0.55 \pm 0.02 \\
5.22 \pm 0.21\end{array}$ & $\begin{array}{r}<0.26 \pm 0.01 \\
1.62 \pm 0.07\end{array}$ & $\begin{array}{l}117.9 \pm 0.4 \\
134.4 \pm 3.6\end{array}$ & $\begin{array}{c}>5.2 \pm 0.3 \\
\ldots\end{array}$ \\
\hline
\end{tabular}

Note. - Column 1: JVN observation epoch; Column 2: component name; Column 3: flux density; Column 4: distance from the origin defined by component C; Column 5: position angle with respect to the origin (measured from north through east); Columns 6, 7, and 8: parameters of Gaussian model: FWHM of major and minor axes and the position angle of the major axis; Column 9: brightness temperature given in the source's rest frame. 
Table 4

Observation summary and map description of VLBA images.

\begin{tabular}{|c|c|c|c|c|c|c|c|c|c|c|}
\hline $\begin{array}{l}\text { Code } \\
\text { (1) }\end{array}$ & Date (e & och) & $\begin{array}{c}\nu_{\mathrm{obs}} \\
{[\mathrm{GHz}]} \\
(3)\end{array}$ & $\begin{array}{c}t_{\mathrm{On}} \\
{[\mathrm{sec}]} \\
(4)\end{array}$ & $\begin{array}{c}\theta_{\text {Bmaj }} \\
{[\text { mas] }} \\
(5)\end{array}$ & $\begin{array}{c}\theta_{\mathrm{Bmin}} \\
{[\mathrm{mas}]} \\
(6)\end{array}$ & $\begin{array}{c}\text { PA } \\
{[\mathrm{deg}]} \\
(7)\end{array}$ & $\begin{array}{c}S_{\text {peak }} \\
{\left[\mathrm{mJy} \mathrm{beam}^{-1}\right]} \\
(8)\end{array}$ & $\begin{array}{c}\sigma \\
{\left[\mathrm{mJy} \mathrm{beam}^{-1}\right]} \\
(9)\end{array}$ & $\begin{array}{l}\text { Contours } \\
\text { (10) }\end{array}$ \\
\hline \multirow[t]{2}{*}{ BB023 } & 1996 May 16 & (1996.374) & 2.269 & 322 & 6.00 & 2.77 & 9.0 & 351 & 2.6 & $n=0,1, \cdots, 11$ \\
\hline & 1996 May 16 & (1996.374) & 8.339 & 322 & 1.73 & 0.73 & 8.7 & 245 & 1.0 & $n=0,1, \cdots, 12$ \\
\hline BK077 & 2003 Oct 9 & (2003.688) & 8.421 & 178 & 2.26 & 1.35 & -16.9 & 334 & 2.5 & $n=0,1, \cdots, 14$ \\
\hline BE042 & 2005 Aug 20 & $(2005.636)$ & 8.420 & 6640 & 1.93 & 0.63 & -2.0 & 204 & 1.3 & $n=0,1, \cdots, 14$ \\
\hline BL156 & 2008 Apr 20 & (2008.303) & 8.392 & 281 & 1.98 & 0.81 & -7.5 & 217 & 1.6 & $n=0,1, \cdots, 14$ \\
\hline BL149 & 2010 Oct 15 & (2010.789) & 15.357 & 2088 & 0.76 & 0.47 & 0.0 & 223 & 0.3 & $n=0,1, \cdots, 15$ \\
\hline
\end{tabular}

Note. - Column 1: VLBA observation code; Column 2: observation date and epoch; Column 3: observation frequency; Column 4: total on-source time; Columns 5, 6, and 7: parameters of the restoring beam: Full-width at half maximum (FWHM) of major and minor axes and the position angle of the major axis; Column 8: peak intensity; Column 9: rms noise level; Column 10: contour levels. The number $n$ corresponds to $-3 \sigma, 3 \sigma \times(\sqrt{2})^{n}$.

Table 5

Model fitting results for archival VLBA observations.

\begin{tabular}{|c|c|c|c|c|c|c|c|c|}
\hline $\begin{array}{c}\text { Epoch } \\
\text { (1) }\end{array}$ & $\begin{array}{c}\nu \\
{[\mathrm{GHz}]} \\
(2)\end{array}$ & $\begin{array}{c}\text { Comp. } \\
\text { (3) }\end{array}$ & $\begin{array}{c}S \\
{[\mathrm{mJy}]} \\
(4)\end{array}$ & $\begin{array}{c}r \\
{[\mathrm{mas}]} \\
(5)\end{array}$ & $\begin{array}{c}\phi \\
{[\mathrm{deg}]} \\
(6)\end{array}$ & $\begin{array}{c}\theta_{\text {maj }} \\
{[\mathrm{mas}]} \\
(7)\end{array}$ & $\begin{array}{c}\theta_{\min } \\
{[\mathrm{mas}]} \\
(8)\end{array}$ & $\begin{array}{c}\text { PA } \\
{[\text { deg] }} \\
(9)\end{array}$ \\
\hline \multirow{2}{*}{1996.374} & 2.269 & $\mathrm{C} 0+\mathrm{D} 2$ & $389 \pm 35$ & 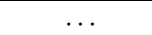 & & $1.33 \pm 0.13$ & $1.00 \pm 0.10$ & $55.9 \pm 0.4$ \\
\hline & & D1 & $168 \pm 10$ & $6.39 \pm 1.31$ & $126.7 \pm 1.2$ & $3.80 \pm 0.38$ & $2.22 \pm 0.22$ & $147.4 \pm 1.7$ \\
\hline \multirow[t]{3}{*}{1996.374} & 8.339 & $\mathrm{C} 0$ & $271 \pm 11$ & & & $0.49 \pm 0.02$ & $0.14 \pm 0.01$ & $125.9 \pm 0.2$ \\
\hline & & D2 & $51 \pm 1$ & $0.58 \pm 0.26$ & $110.6 \pm 0.4$ & $1.37 \pm 0.07$ & $0.13 \pm 0.01$ & $131.8 \pm 3.0$ \\
\hline & & D1 & $40 \pm 1$ & $6.70 \pm 0.32$ & $125.0 \pm 2.4$ & $3.27 \pm 0.16$ & $1.43 \pm 0.07$ & $138.6 \pm 8.2$ \\
\hline \multirow{2}{*}{2003.688} & 8.421 & $\mathrm{C} 0+\mathrm{D} 2$ & $348 \pm 13$ & $\ldots$ & $\ldots$ & $0.52 \pm 0.02$ & $0.35 \pm 0.01$ & $120.5 \pm 0.2$ \\
\hline & & D1 & $40 \pm 1$ & $7.03 \pm 0.31$ & $126.3 \pm 5.4$ & $3.36 \pm 0.13$ & $0.62 \pm 0.03$ & $121.0 \pm 2.4$ \\
\hline \multirow[t]{4}{*}{2005.636} & 8.420 & $\mathrm{C} 0$ & $203 \pm 6$ & & & $0.35 \pm 0.01$ & $0.15 \pm 0.01$ & $148.4 \pm 0.1$ \\
\hline & & D2 & $43 \pm 1$ & $0.50 \pm 0.21$ & $124.6 \pm 0.2$ & $1.48 \pm 0.04$ & $0.15 \pm 0.01$ & $117.8 \pm 1.7$ \\
\hline & & D1b & $13 \pm 1$ & $6.93 \pm 0.20$ & $124.8 \pm 2.0$ & $0.93 \pm 0.02$ & $0.30 \pm 0.01$ & $107.1 \pm 1.5$ \\
\hline & & D1a & $31 \pm 1$ & $7.18 \pm 0.21$ & $125.4 \pm 2.3$ & $5.86 \pm 0.33$ & $1.17 \pm 0.01$ & $127.3 \pm 2.0$ \\
\hline \multirow[t]{4}{*}{2008.303} & 8.392 & $\mathrm{C} 0$ & $179 \pm 5$ & $\ldots$ & $\ldots$ & $0.30 \pm 0.01$ & $0.09 \pm 0.01$ & $137.0 \pm 0.1$ \\
\hline & & D2 & $98 \pm 2$ & $0.57 \pm 0.21$ & $130.7 \pm 0.2$ & $1.35 \pm 0.04$ & $0.22 \pm 0.01$ & $117.6 \pm 0.6$ \\
\hline & & $\mathrm{D} 1 \mathrm{~b}$ & $29 \pm 1$ & $6.86 \pm 0.28$ & $126.3 \pm 5.1$ & $5.11 \pm 0.15$ & $1.09 \pm 0.03$ & $127.0 \pm 3.1$ \\
\hline & & D1a & $12 \pm 1$ & $7.30 \pm 0.21$ & $126.0 \pm 2.4$ & $1.56 \pm 0.05$ & $0.44 \pm 0.01$ & $137.3 \pm 2$ \\
\hline \multirow[t]{4}{*}{2010.789} & 15.357 & $\mathrm{C} 0$ & $193 \pm 4$ & & & $0.18 \pm 0.01$ & $0.04 \pm 0.01$ & $148.5 \pm 0.1$ \\
\hline & & D3 & $48 \pm 4$ & $0.19 \pm 0.19$ & $119.4 \pm 0.1$ & $0.20 \pm 0.01$ & $0.09 \pm 0.01$ & $142.7 \pm 0$. \\
\hline & & D2 & $49 \pm 1$ & $0.67 \pm 0.24$ & $126.3 \pm 0.2$ & $1.97 \pm 0.08$ & $0.29 \pm 0.01$ & $131.5 \pm 2.1$ \\
\hline & & D1 & $19 \pm 1$ & $7.10 \pm 0.27$ & $125.0 \pm 2.4$ & $2.77 \pm 0.11$ & $0.67 \pm 0.03$ & $124.4 \pm 11.8$ \\
\hline
\end{tabular}

Note. - Column 1: VLBA observation epoch; Column 2: observation frequency; Column 3: component name; Column 4: flux density; Column 5: distance from the origin defined by component $\mathrm{C0}$; Column 6: position angle with respect to the origin (measured from north through east); Columns 7, 8, and 9: parameters of Gaussian model: FWHM of major and minor axes and the position angle of the major axis. 